\title{
Cyclic homology and the Macdonald conjectures
}

\author{
Phil Hanlon * \\ Department of Mathematics, California Institute of Technology, Pasadena, CA 91125, USA
}

\begin{abstract}
Summary. Let $A_{+}(k)$ denote the ring $\mathbb{C}[t] / t^{k+1}$ and let $\mathscr{G}$ be a reductive complex Lie algebra with exponents $m_{1}, \ldots, m_{n}$. This paper concerns the Lie algebra cohomology of $\mathscr{G} \otimes A_{+}(k)$ considered as a bigraded algebra (here one of the gradings is homological degree and the other, which we call weight, is inherited from the obvious grading of $\left.\mathscr{G} \otimes A_{+}(k)\right)$. We conjecture that this Lie algebra cohomology is an exterior algebra with $k+1$ generators of homological degree $2 m_{s}+1$ for $s=1,2, \ldots, n$. Of these $k+1$ generators of degree $2 m_{s}+1$, one has weight 0 and the others have weights $(k+1) m_{s}+t$ for $t=1,2, \ldots, k$.

It is shown that this conjecture about the Lie algebra cohomology of $\mathscr{G} \otimes A_{+}(k)$ implies the Macdonald root system conjectures. Next we consider the case that $\mathscr{G}$ is a classical Lie algebra with root system $A_{n}, B_{n}, C_{n}$, or $D_{n}$. It is shown that our conjecture holds in the limit on $n$ as $n$ approaches infinity which amounts to the computation of the cyclic and dihedral cohomologies of $A_{+}(k)$. Lastly we discuss the relevance of this limiting case to the case of finite $n$ in this situation.
\end{abstract}

\section{Section 1. The Macdonald root system conjectures}

Let $\Phi$ be a reduced, finite root system of rank $n$ with basis $\Delta=\left\{\alpha_{1}, \ldots, \alpha_{n}\right\}$. Let $\Phi^{+}$ be the set of positive roots with respect to $\Delta$. Each $\alpha \in \Phi^{+}$can be written uniquely as a non-negative linear combination, $\alpha=\sum c_{i} \alpha_{i}$, of the $\alpha_{i}$. Define the height of $\alpha$ by

$$
h t \alpha=\sum c_{i} .
$$

Recall that there are $n$ positive integers $m_{1}, \ldots, m_{n}$ associated to $\Phi$ called the exponents of $\Phi$. The simplest description of these numbers is in terms of the height function.

* Partially supported by NSF grant number MCS-8401718 and a Bantrell Fellowship

Current address: Department of Mathematics, University of Michigan, Ann Arbor, MI 48109-1003, USA 
Definition 1.1. For each positive integer $i$, let $\Phi_{i}^{+}$denote the subset of $\Phi^{+}$ consisting of all roots of height $i$. Define the exponents $m_{1}, \ldots, m_{n}$ of $\Phi$ by saying that the multiplicity of $i$ as an exponent is $\left|\Phi_{i}\right|-\left|\Phi_{i+1}\right|$.

For each $\alpha \in \mathbb{R}^{n}$, let $e^{\alpha}$ be a formal exponential of $\alpha$. In other words, the $e^{\alpha}$ are a set of commuting indeterminates subject only to the relations

$$
\begin{aligned}
e^{\alpha} e^{\beta} & =e^{\alpha+\beta}, \\
e^{0} & =1 .
\end{aligned}
$$

The following conjectures were originally formulated by I. G. Macdonald. For a more thorough background on these conjectures, the reader should consult [19].

Conjecture 1.2 (Macdonald). Let $\Phi$ be a root system with rank $n$ and exponents $m_{1}, \ldots, m_{n}$ and let $k$ be a positive integer. Let $C(\Phi, k)$ be the polynomial

$$
C(\Phi, k)=\prod_{0 \leqq i \leqq k} \prod_{\alpha \in \Phi^{+}}\left(1-q^{i} e^{\alpha}\right)\left(1-q^{i+1} e^{-\alpha}\right) .
$$

Then the constant term of $C(\Phi, k)$ is

$$
\Pi\left[\begin{array}{c}
(k+1)\left(m_{i}+1\right) \\
k+1
\end{array}\right]_{q}
$$

where $\left[\begin{array}{l}a \\ b\end{array}\right]_{q}$ is the q-binomial coefficient

$$
\left[\begin{array}{l}
a \\
b
\end{array}\right]_{q}=\frac{\left(1-q^{a}\right)\left(1-q^{a-1}\right) \ldots\left(1-q^{a-b+1}\right)}{\left(1-q^{b}\right)\left(1-q^{b-1}\right) \ldots(1-q)}
$$

In the above conjecture "constant term" refers to that part of $C(\Phi, k)$ which is independent of the $e^{\alpha}$.

In the form above, the Macdonald conjectures are known for the following values of $\Phi, k$ and $q$ :

1) $q=1, \Phi=B_{n}, C_{n}, D_{n}$, all $k$ (see [19] or [22])

2) $\Phi=A_{n}$, all $k$, all $q$ (see [2])

3) all $\Phi$, all $q, k=0,1$ (see [19])

4) all $\Phi, k=\infty$, all $q$ (see [7], [20], or [21]).

5) $\Phi=A_{n}, B_{n}, C_{n}, D_{n}$, all $k$, all $q$ as $n \rightarrow \infty$ (see [10]).

In this paper we will start from an equivalent formulation of Conjecture 1.2 also due to Macdonald. A proof of their equivalence can be found in [19].

For this conjecture, let $\mathscr{G}$ be a semisimple complex Lie algebra with root system $\Phi$. We let $\wedge \mathscr{G}$ denote the exterior algebra of $\mathscr{G}$ with $\wedge^{r} \mathscr{G}$ its $r^{\text {th }}$-graded piece. The adjoint action of $\mathscr{G}$ extends naturally to a degree-preserving action of $\mathscr{G}$ on $\wedge \mathscr{G}$.

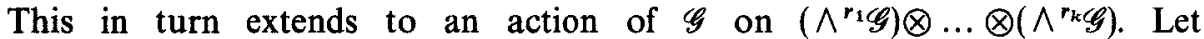
$\left(\left(\wedge^{\left.r_{1} \mathscr{G}\right)} \otimes \ldots \otimes\left(\wedge^{r_{k} \mathscr{G}}\right)\right)^{g}\right.$ denote the subspace of $\left(\wedge^{\left.r_{1} \mathscr{G}\right)} \otimes \ldots \otimes\left(\wedge^{\left.r_{k} \mathscr{G}\right)}\right.\right.$ annihilated by $\mathscr{G}$.

Conjecture 1.3. Let $\mathscr{G}$ be a semisimple complex Lie algebra of rank $n$. Let $\Phi$ be the root system of $\mathscr{G}$ and let $m_{1}, \ldots, m_{n}$ be the exponents of $\Phi$. Then for every positive 
integer $k$,

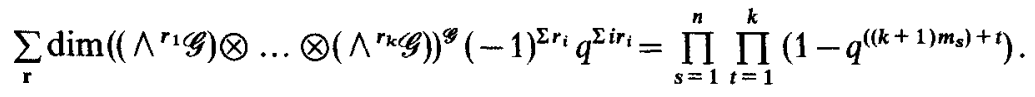

where the sum on the left is over all sequences $\mathbf{r}=\left(r_{1}, \ldots, r_{k}\right)$ of non-negative integers.

Note that the left-hand side of Conjecture 1.3 makes sense for $\mathscr{G}=g 1_{n}(\mathbb{C})$ even though $\mathrm{gl}_{n}(\mathbb{C})$ is not semisimple (and so technically does not have exponents). This problem is remedied by defining the exponents of $\mathrm{gl}_{n}(\mathbb{C})$ to be $0,1, \ldots, n-1$. With this convention, everything stated in this paper for semisimple Lie algebras also holds for $\mathbf{g l}_{n}(\mathbb{C})$.

Recall the notion of Lie algebra cohomology (see for example [4] or [15]). Let $\mathscr{L}$ be a complex Lie algebra. Define $\delta_{r-1}:\left(\wedge^{r-1} \mathscr{L}\right)^{*} \rightarrow\left(\wedge^{r} \mathscr{L}\right)^{*}$ by

$$
(\delta f)\left(x_{1} \wedge \ldots \wedge x_{r}\right)=\sum(-1)^{i+j+1} f\left(\left[x_{i}, x_{j}\right] \wedge x_{1} \wedge \ldots \wedge \hat{x}_{i} \wedge \ldots \wedge \hat{x}_{j} \wedge \ldots \wedge x_{r}\right) .
$$

The Jacobi identity for $\mathscr{L}$ shows that $\delta_{r} \delta_{r-1}=0$. Define the Lie algebra cohomology of $\mathscr{L}$ (with trivial coefficients) to be the graded ring $H L^{*}(\mathscr{L})$ defined by

$$
H L(\mathscr{L})=\left(\operatorname{ker} \delta_{r}\right) /\left(\operatorname{im} \delta_{r-1}\right)
$$

One important result which we will need later is the following theorem (see [3], [12], or [15]).

Theorem 1.4. Let $\mathscr{L}$ be a semisimple, complex Lie algebra of rank $n$ with exponents $m_{1}, \ldots, m_{n}$. Then $H L^{*}(\mathscr{L})$ is isomorphic to an exterior algebra with $n$ generators of degrees $\left(2 m_{i}\right)+1, i=1,2, \ldots, n$.

Suppose next that $\mathscr{L}$ is an $\mathbb{N}$-graded Lie algebra, i.e., $\mathscr{L}$ has a vector space decomposition $\mathscr{L}=\oplus \mathscr{L}_{u}$, and $\left[\mathscr{L}_{u}, \mathscr{L}_{v}\right] \subset \mathscr{L}_{u+v}$. This grading of $\mathscr{L}$ gives a grading of $\wedge \mathscr{L}$ which we call weight. The case we will consider is where $\mathscr{L}=\mathscr{G} \otimes\left(\mathbb{C}[t] / t^{k+1}\right)$. We define the $i^{\text {th }}$-graded piece of this Lie algebra to be $\mathscr{L}_{i}=\mathscr{G} \otimes t^{i}$. It is easy to see that this gives a Lie algebra grading. The exterior algebra $\wedge \mathscr{L}$ is also graded by exterior degree. The two gradings together give an $\mathbb{N}$ bigrading of $\wedge \mathscr{L}$ whose $(d, w)$-graded piece is denoted $\wedge^{d, w} \mathscr{L}$. More explicitely,

$$
\wedge^{d, w} \mathscr{L}=\sum\left\{\left(\wedge^{r_{0}} \mathscr{L}_{0}\right) \otimes\left(\wedge^{r_{1}} \mathscr{L}_{1}\right) \otimes\left(\wedge^{r_{2}} \mathscr{L}_{2}\right) \otimes \ldots\right\}
$$

where the sum is over all $\left(r_{0}, r_{1}, r_{2}, \ldots\right)$ with $\sum r_{i}=r$ and $\sum i r_{i}=w$.

We can now state a set of conjectures in terms of relative cohomology of Lie algebras which imply the Macdonald root system conjectures. The idea to restate the conjectures in terms of Lie algebra cohomology did not originate with this author. It was observed by Garland and Lepowsky that Conjecture 1.2 has a stronger homological form which they proved when $k$ is infinity (see [7]). We will state a strengthened version of Conjecture 1.3 which we henceforth refer to as the strong Macdonald conjectures.

Conjecture 1.5. (The strong Macdonald root system conjectures.) Let $\mathscr{G}$ be a semisimple complex Lie algebra of rank $n$ with exponents $m_{1}, \ldots, m_{n}$. Let $k$ be a 
positive integer and let $A_{+}(k)$ be the ring $\mathbb{C}[t] /\left(t^{k+1}\right)$. Let $\mathscr{L}$ be the Lie algebra $\mathscr{G} \otimes A_{+}(k)$. Then the cohomology of $\mathscr{L}$ is an exterior algebra with $n(k+1)$ generators. For each $s$, there are $k+1$ generators of degree $\left(2 m_{s}\right)+1$. One of these $k+1$ generators has weight 0 , and the other $k$ have weights $(k+1) m_{s}+t$ for $t=1,2, \ldots, k$.

Before proceeding, we pause to show that the strong Macdonald conjectures imply the previously stated Macdonald conjectures.

Proposition 1.6. Let $\mathscr{G}$ be a semisimple complex Lie algebra. If the strong Macdonald conjectures hold for the Lie algebra $\mathscr{G} \otimes A_{+}(k)$, then Conjectures 1.3 holds for $\mathscr{G}$ and $k$.

Proof. Assume Conjecture 1.5 holds for $\mathscr{L}=\mathscr{G} \otimes \mathbb{C}[t] / t^{k+1}$. Let $\mathscr{G}_{0}$ denote the subalgebra $\mathscr{G} \otimes 1$ of $\mathscr{L}$. By Proposition (v) on page 116 of Guichardet [9], we have the following isomorphism of bigraded algebras:

$$
H^{* *}(\mathscr{L})=H^{* 0}\left(\mathscr{G}_{0}\right) \otimes H^{* *}(\overline{\mathscr{L}})^{\mathscr{G}_{0}}
$$

where $\mathscr{L}$ is the ideal $\mathscr{G} \otimes\left(t \mathbb{C}[t] / t^{k+1}\right)$ in $\mathscr{L}$. Since the strong Macdonald conjectures hold for $\mathscr{L}, H^{* *}(\overline{\mathscr{L}})^{\mathscr{G}_{0}}$ is an exterior algebra with $k$ generators of degree $2 m_{s}+1$ for each $s=1,2, \ldots, n$. Moreover these $k$ generators have weights $(k+1) m_{s}+t$ for $t=1,2, \ldots, k$. Thus

$$
\sum_{r, w} \operatorname{dim}\left(H^{r, w}(\mathscr{L})^{\mathscr{G}_{0}}\right)(-1)^{r} q^{w}=\prod_{s=1}^{n} \prod_{t=1}^{k}\left(1-q^{(k+1) m_{s}+t}\right) .
$$

By complete reducibility of representations of $\mathscr{G}, H^{*, w}(\overline{\mathscr{L}})^{\mathscr{G}_{0}}$ is the cohomology of the graded complex

$$
\wedge^{0, w}(\overline{\mathscr{L}})^{\mathscr{G}_{0}} \rightarrow \wedge^{1, w}(\overline{\mathscr{L}})^{\mathscr{G}_{0}} \rightarrow \wedge^{2, w}(\overline{\mathscr{L}})^{\mathscr{G}_{0}} \rightarrow \ldots
$$

Computing the Euler characteristic of this complex we get, for each $w$,

$$
\sum_{r} \operatorname{dim}\left(\wedge^{r, w}(\overline{\mathscr{L}})^{\mathscr{G}_{0}}\right)(-1)^{r}=\sum_{r} \operatorname{dim}\left(H^{r, w}(\overline{\mathscr{L}})^{\mathscr{S}_{0}}\right)(-1)^{r}
$$

Substituting in equation (1.1) we obtain,

$$
\sum_{r, w} \operatorname{dim}\left(\wedge^{r, w}(\mathscr{L})^{\mathscr{S}_{0}}\right)(-1)^{r} q^{w}=\prod_{s=1}^{n} \prod_{t=1}^{k}\left(1-q^{(k+1) m_{s}+t}\right) .
$$

Write $\overline{\mathscr{L}}=\mathscr{G}_{1} \oplus \ldots \oplus \mathscr{G}_{k}$ where $\mathscr{G}_{i}=\mathscr{G} \otimes t^{i}$. Then

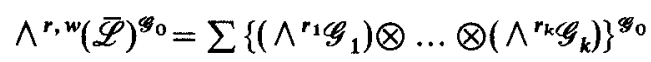

where the sum is over all $\left(r_{1}, \ldots, r_{k}\right)$ with $\sum r_{i}=r$ and $\sum i r_{i}=w$. Thus (1.2) is equivalent to

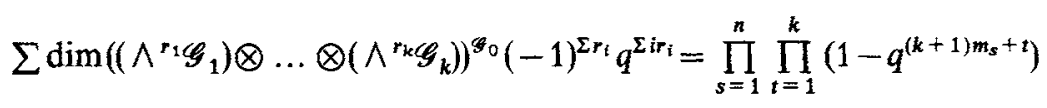

which is exactly Conjecture 1.3 . 
The rest of this paper is devoted to proving something close to the strong Macdonald conjectures for the classical Lie algebras. In the case of the classical Lie algebras, $\mathscr{G}_{n}=\mathrm{sl}_{n}(\mathbb{C}), o_{n}(\mathbb{C})$, or $\operatorname{sp}_{2 n}(\mathbb{C})$, the complex $\left(C_{n}{ }^{d, w}, \delta\right)$ for computing the cohomology of $\mathscr{G}_{n} \otimes A_{+}(k)$ approaches a limit as $n$ approaches infinity. That is to say, there is a bigraded complex $\left(C^{d, w}, \delta\right)$ with the property that $\left(C_{n}^{d, w}, \delta\right)$ is a quotient of $C^{d, w}$ by a $\delta$-invariant subspace $J_{n}{ }^{d, w}$, and $J_{n}{ }^{d, w}$ is zero for $n$ large with respect to $d$. As $C_{n}^{d, w}=C^{d, w} / J_{n}{ }^{d, w}$, there is a natural homomorphism $\pi_{n}: H^{d, w}(C)$ $\rightarrow H^{d, w}\left(\mathscr{G}_{n} \otimes A_{+}(k)\right)$. Thus part of the cohomology of $\mathscr{G}_{n} \otimes A_{+}(k)$ is projected from the $n$ equals infinity complex.

We will compute this image $\pi_{n}\left(H^{*, *}(C)\right.$ ) (in Theorems 4.9 and 4.12 ) and show that it is an exterior algebra with $n(k+1)$ generators of degrees $\left(2 m_{s}\right)+1$ with weights 0 and $\left((k+1) m_{s}\right)+t$, for $s=1,2, \ldots, n$ and $t=1,2, \ldots, k$. The strong Macdonald conjectures are verified for the classical Lie algebras if it is shown that $\pi_{n}$ is onto. Given Theorems 4.9 and 4.12 , there are several weaker conditions that will imply the strong Macdonald conjectures. We summarize these in the following proposition.

Proposition 1.7. Let $\mathscr{G}_{n}$ be a semisimple, complex Lie algebra with root system $A_{n}$, $B_{n}, C_{n}$, or $D_{n}$. Then any of the following three conditions imply the strong Macdonald conjectures for the Lie algebra $\mathscr{G}$ and the positive integer $k$.

C1) The map $\pi_{n}$ is onto.

C2) The cohomology ring $H^{*}\left(\mathscr{G}_{n} \otimes A_{+}(k)\right)$ is an exterior algebra with generators of odd degree.

C3) The cohomology of the Lie algebra $\mathscr{G}_{n} \otimes A_{+}(k)$ has dimension $2^{n(k+1)}$.

Proof. It is obvious that $\mathrm{C} 1$ implies Conjecture 1.5 (given the result mentioned above). Suppose C2 holds. Using Proposition (v) from Guichardet [8, p. 116] we have

$$
\sum \operatorname{dim}\left(H^{d, w}\left(\overline{\mathscr{G}_{n} \otimes A_{+}(k)}\right)^{\mathscr{G}_{n}}\right)(-1)^{d} q^{w}=\left(1-q^{u_{1}}\right)\left(1-q^{u_{2}}\right) \ldots\left(1-q^{u_{N}}\right)
$$

where $N$ is the number of generators of the exterior algebra $\left.H^{*}\left(\overline{\mathscr{G}_{n} \otimes A_{+}(k)}\right)^{\mathscr{g}_{n}}\right)$. If $N$ is less than or equal to $n k$, then we are done by C1. So assume $N>n k$. Reversing the integration argument given by Macdonald (see [19]) we obtain an expression $E(q)$ for the right hand side of Conjecture 1.2 from the product $\Pi\left(1-q^{u_{i}}\right)$. Since $N$ is greater than $n k, E(1)=0$. But this contradicts the weak Macdonald conjectures at $q=1$ which are known to hold for the classical root systems.

Lastly suppose that C3) holds. Since the image of $\pi_{n}$ has dimension $2^{n(k+1)}$ it must be that $\pi_{n}$ is onto which completes the proof.

Condition C3 of Proposition 1.7 leads to an interesting deformation-theoretic interpretation of the strong Macdonald conjectures. For each complex number $z$, let $L_{z}$ be the Lie algebra

$$
L_{z}=\mathscr{G} \otimes\left(\mathbb{C}[t] /\left(t^{(k+1)}-z\right)\right)
$$

It is easy to check that $L_{z}$ is isomorphic to a (Lie algebra) direct sum of $k+1$ copies of $\mathscr{G}$ for $z$ not equal to 0 . So for nonzero $z$, the Lie algebra cohomology of $L_{z}$ is isomorphic to the tensor product of $k+1$ copies of $H^{*}(\mathscr{G})$. The structure of 
$L_{0}=\mathscr{G} \otimes A_{+}(k)$ is very different. The strong Macdonald conjectures say that even though the Lie algebra structure of $L_{z}$ changes at $z=0$, the cohomology remains constant.

\section{Section 2: Cyclic and dihedral homology}

Let $A$ be any associative $\mathbb{C}$-algebra and let $M$ be an $A$-bimodule. By definition, $H_{*}(A ; M)$ is the space of derived functors of $(-) \otimes_{A \otimes A} M$ evaluated on $A$. One particular complex for computing $H_{*}(A ; M)$ is $\left(C_{n}, \partial\right)$ where

$$
C_{n}=M \otimes A \otimes A \otimes \ldots \otimes A \quad(n \text { copies of } A)
$$

and

$$
\begin{aligned}
& \partial\left(m \otimes a_{1} \otimes \ldots \otimes a_{n}\right)=\left(m a_{1}\right) \otimes a_{2} \otimes \ldots \otimes a_{n} \\
& \quad+\left\{\Sigma(-1)^{i} m \otimes a_{1} \otimes \ldots \otimes\left(a_{i} a_{i+1}\right) \otimes \ldots \otimes a_{n}\right\} \\
& \quad+(-1)^{n}\left(a_{n} m\right) \otimes a_{1} \otimes \ldots \otimes a_{n-1} .
\end{aligned}
$$

If $M=\mathbb{C}$ is the trivial $A$-bimodule, then the above complex is the Bar complex for computing $H_{*}(A ; \mathbb{C})$ and if $M=A$ with module structure being the regular representation of $A$ on itself, then the above complex is the Hochschild complex for computing $H_{*}(A ; A)$. We denote the differentials in the Bar complex and the Hochschild complex by $b^{\prime}$ and $b$ respectively.

Consider the case where $M$ is itself a $\mathbb{C}$-algebra, where $A$ is commutative and where the left and right actions of $A$ on $M$ are identical. Define a product $\circ$ : $C_{n} \times C_{p} \rightarrow C_{n+p}$ by

$$
\begin{gathered}
\left(m \otimes a_{1} \otimes \ldots \otimes a_{n}\right) \circ\left(m^{\prime} \otimes a_{n+1} \otimes \ldots \otimes a_{n+p}\right) \\
\quad=\sum \operatorname{sgn}(\sigma)\left(m m^{\prime}\right) \otimes a_{\sigma 1} \ldots \otimes a_{\sigma(n+p)}
\end{gathered}
$$

where the sum is over all $\sigma \in S_{n+p}$ with the property that

$$
\sigma 1<\sigma 2<\ldots<\sigma n \text { and } \sigma(n+1)<\ldots<\sigma(n+p) .
$$

It is easy to check, for $\alpha \in C_{n}$ and $\beta \in C_{p}$, that

$$
\partial(\alpha \circ \beta)=(\partial \alpha \circ \beta)+(-1)^{n}(\alpha \circ \partial \beta) \text {. }
$$

In particular, $\circ$ induces a product $\circ: H_{n}(A ; M) \times H_{p}(A ; M) \rightarrow H_{n+p}(A ; M)$. This product is called the shuffle product.

Recall the $\mathbb{C}$-algebra $A_{+}(k)=\mathbb{C}[t] /\left(t^{k+1}\right)$. We will compute certain shuffle products in the case $A=M=A_{+}(k)$. Although these computations are not strictly necessary for what follows, we do them to simplify the upcoming arguments and to display explicit representatives for certain homology and cohomology classes. In what follows we use the notation $T^{r}(V)$ to denote the $r^{\text {th }}$ tensor power of a vector space $V$. 
Definition 2.1. Define $x \in T^{2}\left(A_{+}(k)\right)$ and $y_{(i)} \in T^{3}\left(A_{+}(k)\right)$ by:

$$
\begin{gathered}
x=1 \otimes t \\
y_{(i)}=1 \otimes t^{k} \otimes t+t \otimes t^{k-1} \otimes t+\ldots+t^{i-1} \otimes t^{k-i+1} \otimes t .
\end{gathered}
$$

Lemma 2.2. For $u$ and $v$ integers, define $\alpha_{u}(v)$ to be 1 if $v$ is less than or equal to $u$ and 0 otherwise. Then

A) $\left.t^{s} \circ y_{(k)}{ }^{u}=\sum \alpha_{k}\left(\sum a_{i}\right)+s\right) t^{\left(\Sigma a_{i}\right)+s} \otimes t^{k-a_{1}} \otimes t \otimes \ldots \otimes t^{k-a_{u}} \otimes t$

and

B) $t^{s} \circ x \circ y_{(k)}{ }^{u}=\sum \alpha_{k}\left(\left(\sum a_{i}\right)+s\right) t^{\left(\sum a_{i}\right)+s} \otimes t \otimes t^{k-a_{1}} \otimes \ldots \otimes t^{k-a_{u}} \otimes t$,

where $t^{u}$ refers to the ordinary $u^{\text {th }}$ power of $t$ and $y_{(k)}{ }^{u}$ refers to the $u^{\text {th }}$ shuffle power of $y_{(k)}$.

Claim. $y^{u}=\sum t^{\sum a_{i}} \otimes t^{k-a_{1}} \otimes t \otimes \ldots \otimes t^{k-a_{u}} \otimes t$ where the sum is over all $\left(a_{1}, \ldots, a_{u}\right)$ with $\sum a_{i} \leqq k$.

To begin the proof of this claim, write $y^{u}$ out as

$$
y^{u}=\left(\sum t^{a_{1}} \otimes t^{k-a_{1}} \otimes t\right) \circ\left(\sum t^{a_{2}} \otimes t^{k-a_{2}} \otimes t\right) \circ \ldots \circ\left(\sum t^{a_{u}} \otimes t^{k-a_{u}} \otimes t\right) .
$$

Each choice of $\left(a_{1}, \ldots, a_{u}\right)$ gives rise to a number of terms in the shuffle product. Each of these terms begins with $t^{\Sigma a_{i}}$, hence each of these terms is 0 unless $\sum a_{i} \leqq k$.

Choose $\left(a_{1}, \ldots, a_{u}\right)$ with $\sum a_{i} \leqq k$. We consider the corresponding term (2.1) in the shuffle power $y^{u}$,

$$
\left(t^{a_{1}} \otimes t^{k-a_{1}} \otimes t\right) \circ\left(t^{a_{2}} \otimes t^{k-a_{2}} \otimes t\right) \circ \ldots \circ\left(t^{a_{u}} \otimes t^{k-a_{u}} \otimes t\right) .
$$

Let $i$ and $i^{\prime}$ correspond to $t^{k-a_{i}}$ and $t$, i.e., the second and third tensor positions in the $i^{\text {th }}$ factor of (2.1). The full shuffle product in (2.1) is a sum of terms indexed by the permutations $\sigma$ of $1,1^{\prime}, 2,2^{\prime}, \ldots, u, u^{\prime}$ satisfying $\sigma(i)<\sigma\left(i^{\prime}\right)$ for all $i$. We call such a permutation admissible, and let $\tau_{\sigma}$ denote the term in (2.1) indexed by $\sigma$.

Let $\sigma$ be an admissible permutation. We say $i^{\prime}$ and $j^{\prime}$ are consecutive primed numbers with respect to $\sigma$ if $\sigma\left(i^{\prime}\right)$ immediately precedes $\sigma\left(j^{\prime}\right)$ in the order $1<1^{\prime}<\ldots<u<u^{\prime}$. Note that if $\pi$ is the permutation obtained from $\sigma$ by interchanging $\sigma\left(i^{\prime}\right)$ and $\sigma\left(j^{\prime}\right)$, then $\pi$ is also an admissible permutation. We say that two admissible permutations $\sigma$ and $\pi$ are equivalent if $\pi$ is obtained from $\sigma$ by a series of interchanges of consecutive primed numbers.

Let $\sigma$ be an admissible permutation written out into groups of consecutive nonprimed and primed numbers, with $w_{1}, v_{1}, w_{2}, v_{2}, \ldots, w_{u}, v_{u}$ the sizes of the groups. Then the size of the equivalence class containing $\sigma$ is $v_{1} ! v_{2} ! \ldots v_{u} !$, there being one admissible permutation equivalent to $\sigma$ for each choice

$$
\left(\alpha_{1}, \alpha_{2}, \ldots, \alpha_{u}\right) \in \operatorname{Sym}_{v_{1}} \times \operatorname{Sym}_{v_{2}} \times \ldots \times \operatorname{Sym}_{v_{u}}
$$

of a permutation in each group of consecutive primed numbers. Also if $\pi$ is equivalent to $\sigma$ via $\left(\alpha_{1}, \ldots, \alpha_{u}\right)$ then $\operatorname{sgn}(\pi)=\operatorname{sgn}(\sigma)\left(\Pi \operatorname{sgn}\left(\alpha_{i}\right)\right)$. 
Thus we have that

$$
\sum \operatorname{sgn}(\pi)=\left\{\begin{array}{l}
\operatorname{sgn}(\sigma) \text { if } v_{1}=v_{2}=\ldots=v_{u}=1 \\
0 \text { otherwise }
\end{array}\right.
$$

Lastly note that if $\sigma$ and $\pi$ are equivalent, admissible, permutations then the terms in the shuffle product (2.1) indexed by $\sigma$ and $\pi$ are equal. So there is no contribution to (2.1) except by equivalence classes of size one. But it is easy to see that the only admissible permutation which is equivalent to no others is the identity and so the shuffle product (2.1) consists of only one term, this being

$$
t^{\Sigma a_{i}} \otimes t^{k-a_{1}} \otimes t \otimes \ldots \otimes t^{k-a_{u}} \otimes t
$$

This proves the claim.

A similar argument shows that

$$
(1 \otimes t) \circ\left(t^{\Sigma a_{i}} \otimes t^{k-a_{1}} \otimes t \otimes \ldots \otimes t^{k-a_{u}} \otimes t\right)
$$

also consists of only one term, this being

$$
t^{\Sigma a_{i}} \otimes t \otimes t^{k-a_{1}} \otimes t \otimes \ldots \otimes t^{k-a_{u}} \otimes t .
$$

Lemma 2.2 now follows immediately.

Assume now that $M$ is a filtered $A$-bimodule and let $E^{0}(M)$ denote the associated graded object. Then there is a spectral sequence converging to $H_{d}(A ; M)$ with $E^{1}{ }_{d, q}=H_{d}\left(A ; E_{q}^{0}(M)\right)$. Each term $E^{r}$ is bigraded, $E^{r}=\oplus E_{d, q}^{r}$ by homological degree $d$, and filtration degree $q$. The differential $\partial_{r}$ of $E^{r}$ maps $E_{d, q}^{r}$ to $E_{d-r, q+r-1}^{r}$.

This situation arises when $A$ is an augmented algebra with augmentation map $\varepsilon: A \rightarrow \mathbb{C}$ and augmentation ideal $A=\operatorname{ker} \varepsilon$. In this case, $M$ can be given the $A$-adic filtration which makes $E^{0}(M)$ into a trivial $A$-module. So the $E^{1}$ term simplifies to $E_{d, q}^{1}=M \otimes H_{d}(A ; \mathbb{C})$. In the more specialized situation where $M=A$ with the obvious bimodule structure, and where $A$ is an $\mathbb{N}$-graded, commutative $\mathbb{C}$-algebra, the grading of $A$ induces a grading of $H_{*}(A ; N)$ for any $N$, which we call weight. The above spectral sequence becomes trigraded, $E_{d, q, w}^{r}$ with $d$ being homological degree, $q$ being filtration degree, and $w$ being weight. The differential $\partial_{r}$ preserves weight and the sequence $E_{d, q, w}^{r}$ converges to $H_{d, w}(A ; A)$. We record these wellknown facts in a lemma for future reference.

Lemma 2.3. Let $A$ be an $\mathbb{N}$-graded, commutative $\mathbb{C}$-algebra. Then there is a trigraded spectral sequence

$$
\left\{E_{\mathrm{d}, q, w}^{r}, \partial_{r}\right\} \Rightarrow H_{d, w}(A ; A)
$$

with $E^{1}$ term $E_{d, q, w}^{1}=A_{q} \otimes H_{d, w}(A ; \mathbb{C})$.

Theorem 2.4. Let notation be as above. Then

$$
\operatorname{dim}\left(H_{0, w}\left(A_{+}(k) ; A_{+}(k)\right)\right)= \begin{cases}1 & \text { if } w \leqq k \\ 0 & \text { otherwise }\end{cases}
$$


For $d>0$,

$\operatorname{dim}\left(H_{d, w}\left(A_{+}(k) ; A_{+}(k)\right)\right)=\left\{\begin{array}{lll}1 & \text { if } d=2 e \text { and }(k+1) e<w<(k+1)(e+1) \\ 1 & \text { if } d=2 e+1 \text { and }(k+1) e<w<(k+1)(e+1) \\ 0 & \text { otherwise. }\end{array}\right.$

Moreover let $y_{(k)}=\left(t \otimes t^{k} \otimes t\right)+\left(t^{2} \otimes t^{k-1} \otimes t\right)+\ldots+\left(t^{k} \otimes t \otimes t\right)$. Then a set of homology representatives for the nonzero homology groups $H_{d, w}\left(A_{+}(k) ; A_{+}(k)\right)$ are given below:

$(d, w)$

representative for $H_{d, w}\left(A_{+}(k) ; A_{+}(k)\right)$

$(0, w) 0 \leqq w \leqq k$

$t^{w}$

$(1, w) 1 \leqq w \leqq k$

$t^{w-1} \otimes t$

$(2 e,(k+1) e+s) 1 \leqq s \leqq k$

$t^{s} \circ y \circ y \circ \ldots \circ y$

$(2 e+1,(k+1) e+s) 1 \leqq s \leqq k$

$t^{s-1} \circ(1 \otimes t) \circ y \circ \ldots \circ y$.

Here $u \circ v$ denotes the shuffle product of $u$ and $v$. In each of the last two rows, the representatives end with the $e^{\text {th }}$ shuffle power of $y$.

Proof (Steve Mitchell). Our first step is to compute $H_{*}\left(A_{+}(k) ; \mathbb{C}\right)$. Recall that

$$
H_{*}\left(A_{+}(k) ; \mathbb{C}\right)=\operatorname{Tor}^{A_{+}(k)}(\mathbb{C}, \mathbb{C})
$$

and note that there exists the following free resolution:

$$
\ldots \stackrel{f_{k}}{\longrightarrow} A_{+}(k) \stackrel{f_{1}}{\longrightarrow} A_{+}(k) \stackrel{f_{k}}{\longrightarrow} A_{+}(k) \stackrel{f_{1}}{\longrightarrow} A_{+}(k) \stackrel{\varepsilon}{\longrightarrow} \mathbb{C} \longrightarrow 0
$$

In (2.2), the map $f_{k}$ is multiplication by $t^{k}$, the map $f_{1}$ is multiplication by $t$, and the map $\varepsilon$ is evaluation at 0 .

Upon applying the functor $\mathbb{C} \otimes A_{+}(k)$ to $(2.2)$ we obtain the complex

$$
\ldots \rightarrow \mathbb{C} \rightarrow \mathbb{C} \rightarrow \mathbb{C} \rightarrow 0
$$

with all maps 0 . Thus $H_{d}\left(A_{+}(k) ; \mathbb{C}\right)$ has dimension 1 for all $d$.

Using the usual bar resolution for computing $H_{*}\left(A_{+}(k) ; \mathbb{C}\right)$ one can show that representatives for $H_{d}\left(A_{+}(k) ; \mathbb{C}\right)$ are given by:

$$
\begin{array}{ll}
t^{k} \otimes t \otimes t^{k} \otimes \ldots \otimes t^{k} \otimes t & \text { for } \quad d \text { odd } \\
t \otimes t^{k} \otimes t \otimes \ldots \otimes t^{k} \otimes t & \text { for } \quad d \text { even } .
\end{array}
$$

Let $x=t$ and $y=t^{k} \otimes t$ be the representatives given above for $H_{1}\left(A_{+}(k) ; \mathbb{C}\right)$ and $H_{2}\left(A_{+}(k) ; \mathbb{C}\right)$. By an argument similar to the one given earlier to prove Lemma 2.2 , one can show that the representative given above is exactly:

$$
\begin{array}{ll}
y \circ y \circ \ldots \circ y & \text { if } d \text { is odd } \\
x \circ y \circ y \circ \ldots \circ y & \text { if } d \text { is even. }
\end{array}
$$


Here $\circ$ denotes shuffle product. So the ring structure of $H_{*}\left(A_{+}(k) ; \mathbb{C}\right)$ is given by

$$
H_{*}\left(A_{+}(k) ; \mathbb{C}\right)=\operatorname{Ext}(x) \otimes \operatorname{Sym}(y)
$$

where Ext and Sym denote exterior and symmetric algebras respectively.

Now to compute $H_{*, *}\left(A_{+}(k) ; A_{+}(k)\right)$ we use the spectral sequence of Lemma 2.3. The $E^{1}$ term is

$$
E^{1}=A_{+}(k) \otimes \operatorname{Ext}(x) \otimes \operatorname{Sym}(y),
$$

and the filtration degree of $t^{r} \otimes \operatorname{Ext}(x) \otimes \operatorname{Sym}(y)$ is $r$. Note that the filtration stops at degree $k$ so the spectral sequence must collapse at $E^{k+1}$. In other words,

$$
E^{k+1}=H_{*, *}\left(A_{+}(k) ; A_{+}(k)\right) \text {. }
$$

We claim that

$$
E^{i}=A_{+}(k) \otimes \operatorname{Ext}(x) \otimes \operatorname{Sym}\left(y_{(i)}\right)
$$

for $1 \leqq i \leqq k$. The proof is by induction on $i$.

Recall $y_{(i)}$ which was defined by

$$
y_{(i)}=1 \otimes t^{k} \otimes t+t \otimes t^{k-1} \otimes t+\ldots+t^{i-1} \otimes t^{k-i+1} \otimes t .
$$

We have

$$
\begin{aligned}
\partial_{i-1}\left(y_{(i)}\right)= & \left(t^{k} \otimes t+t \otimes t^{k}\right)+\left(t^{k} \otimes t-t \otimes t^{k}+t^{2} \otimes t^{k-1}\right) \\
& +\left(t^{k} \otimes t-t^{2} \otimes t^{k-1}+t^{3} \otimes t^{k-2}\right)+\ldots \\
& +\left(t^{k} \otimes t-t^{i-1} \otimes t^{k-i+2}+t^{i} \otimes t^{k-i+1}\right) \\
= & i\left(t^{k} \otimes t\right)+\left(t^{i} \otimes t^{k-i+1}\right) \\
= & 0 \text { in } \quad E^{i-1} .
\end{aligned}
$$

Also $y_{(i)}=y_{(i-1)}$ in $E^{i-1}$. So $y_{(i)}$ is a lifting of $y_{(i-1)}$ to the kernal of $\partial_{i-1}$. It is clear that $\partial_{i-1} x=0$ and so it follows easily that $A_{+}(k) \otimes \operatorname{Ext}(x) \otimes \operatorname{Sym}\left(y_{(i-1)}\right)$ in $E^{i-1}$ lifts to $A_{+}(k) \otimes \operatorname{Ext}(x) \otimes \operatorname{Sym}\left(y_{(i)}\right)$ in $E^{i}$.

By induction on $i$ we have that $E^{k}=A_{+}(k) \otimes \operatorname{Ext}(x) \otimes \operatorname{Sym}\left(y_{(k)}\right)$. Now consider $\partial_{k}$ acting on $E^{k}$.

First we compute $\partial_{k}\left(t^{j} \circ x \circ y_{(k+1)}{ }^{u}\right)$. Using Lemma 2.2,

$$
\begin{aligned}
\partial_{k}\left(t^{j} \circ x \circ y_{(k+1)}{ }^{u}\right) & =\partial_{k}\left(\sum t^{\left(\Sigma a_{i}\right)+j} \otimes t \otimes t^{k-a_{1}} \otimes \ldots \otimes t^{k-a_{u}} \otimes t\right) \\
& =\mathscr{A}-\mathscr{B}+\mathscr{C}-\mathscr{D}
\end{aligned}
$$

where $\mathscr{A}=\sum \alpha_{k}\left(\left(\sum a_{i}\right)+j+1\right) t^{\left(\Sigma a_{i}\right)+j+1} \otimes t^{k-a_{1}} \otimes \ldots \otimes t^{k-a_{u}} \otimes t$

$$
\begin{aligned}
& \mathscr{B}=\sum \alpha_{k}\left(k-a_{1}+1\right) t^{\left(\Sigma a_{i}\right)+j} \otimes t^{k-a_{1}+1} \otimes \ldots \otimes t^{k-a_{u}} \otimes t \\
& \mathscr{C}=\sum \alpha_{k}\left(k-a_{u}+1\right) t^{\left(\Sigma a_{i}\right)+j} \otimes t \otimes t^{k-a_{1}} \otimes \ldots \otimes t \otimes t^{k-a_{u}+1}
\end{aligned}
$$

and

$$
\mathscr{D}=\sum \alpha_{k}\left(\left(\sum a_{i}\right)+j+1\right) t^{\left(\Sigma a_{i}\right)+j+1} \otimes t \otimes \ldots \otimes t \otimes t^{k-a_{u}}
$$


Note that there is an exact cancellation of terms with the $\left(a_{1}, \ldots, a_{u}\right)$ term from $\mathscr{A}$ cancelling the $\left(a_{1}+1, a_{2}, \ldots, a_{u}\right)$ term from $\mathscr{B}$ and the $\left(a_{1}, \ldots, a_{u}\right)$ term from $\mathscr{C}$ cancelling the $\left(a_{1}, \ldots, a_{u}-1\right)$ term from $\mathscr{D}$.

Hence $\partial_{k}$ acts trivially on $A_{+}(k) \otimes x \otimes \operatorname{Sym}\left(y_{(k+1)}\right)$.

Next consider $\partial_{k}$ on $A_{+}(k) \otimes 1 \otimes \operatorname{Sym}\left(y_{(k+1)}\right)$. Again using Lemma 2.2, we have

where

$$
\begin{aligned}
\partial_{k}\left(t^{j} \circ y_{(k+1)}{ }^{u}\right) & =\partial_{k}\left(\sum t^{\left(\Sigma a_{i}\right)+j} \otimes t^{k-a_{1}} \otimes t \otimes \ldots \otimes t^{k-a_{u}} \otimes t\right) \\
& =\mathscr{A}+\mathscr{B}-\mathscr{C}
\end{aligned}
$$

$$
\begin{aligned}
& \mathscr{A}=\sum \alpha_{k}\left(\left(\sum a_{i}\right)+j+k-a_{1}\right) t^{\left.\left(\Sigma a_{i}\right)+j+k-a_{1}\right)} \otimes t \otimes \ldots \otimes t^{k-a_{u}} \otimes t \\
& \mathscr{B}=\sum \alpha_{k}\left(k-a_{u}+1\right) t^{\left(\Sigma a_{i}+j\right)} \otimes t^{k-a_{1}} \otimes t \otimes \ldots \otimes t \otimes t^{k-a_{u}+1}
\end{aligned}
$$

and

$$
\mathscr{C}=\sum \alpha_{k}\left(\left(\sum a_{i}\right)+j+1\right) t^{\left(\left(\Sigma a_{i}\right)+j+1\right)} \otimes t^{k-a_{1}} \otimes t \otimes \ldots \otimes t \otimes t^{k-a_{u}} .
$$

As above, $\mathscr{B}-\mathscr{C}=0$ and so $\left.\partial_{k}\left(t^{j} \circ y_{(k+1)}\right)^{u}\right)=\mathscr{A}$. Note that

So,

$$
\alpha_{k}\left(\left(\sum a_{i}\right)+j+k-a_{1}\right)= \begin{cases}1 & \text { if } j=a_{2}=a_{3}=\ldots=a_{u}=0 \\ 0 & \text { otherwise. }\end{cases}
$$

$$
\partial_{k}\left(t^{j} \circ y_{(k+1)}{ }^{u}\right)=\left\{\begin{array}{lll}
(k+1)\left(t^{k} \otimes t \otimes t^{k} \otimes \ldots \otimes t^{k} \otimes t\right) & \text { if } j=0 \\
0 & \text { if } j>0
\end{array}\right.
$$

On the other hand,

$$
\begin{aligned}
t^{k} \circ x \circ y_{(k+1)}^{u-1} & =\sum \alpha_{k}\left(k+\left(\sum a_{i}\right)\right) t^{k+\left(\sum a_{i}\right)} \otimes t \otimes t^{k-a_{1}} \otimes \ldots \otimes t^{k-a_{u}} \otimes t \\
& =t^{k} \otimes t \otimes t^{k} \otimes \ldots \otimes t^{k} \otimes t .
\end{aligned}
$$

Thus $\partial_{k}$ is 0 on $A_{+}(k) \otimes 1 \otimes y_{(k+1)}{ }^{\prime}$ except on the one dimensional space $\left\langle 1 \otimes 1 \otimes y_{(k+1)}{ }^{u}\right\rangle$ which it maps onto $\left\langle t^{k} \otimes x \otimes y_{(k+1)}{ }^{u-1}\right\rangle$.

We've shown that as an $A_{+}(k)$-module, $H_{*, *}\left(A_{+}(k) ; A_{+}(k)\right)$ is cyclic in each dimension with $H_{0, *}\left(A_{+}(k) ; A_{+}(k)\right)=A_{+}(k)$ and $H_{d, *}\left(A_{+}(k) ; A_{+}(k)\right)=A_{+}(k) /\left(t^{k}\right)$ for $d>0$. Moreover, multiplication by $x$ is an isomorphism between $H_{2 s, *}\left(A_{+}(k) ; A_{+}(k)\right)$ and $H_{2 s+1, *}\left(A_{+}(k) ; A_{+}(k)\right)$. Explicit generators are as given in the statement of this theorem.

Let $s_{d}$ be the antisymmetric cyclic shift map on $T^{d}(A)$ defined by

$$
s_{d}\left(a_{1} \otimes \ldots \otimes a_{d}\right)=(-1)^{d-1}\left(a_{d} \otimes a_{1} \otimes \ldots \otimes a_{d-1}\right) .
$$

Note that $s_{d}$ preserves both degree and weight.

Also define the map $\sigma_{d}: T^{d}(A) \rightarrow T^{d}(A)$ by

$$
\sigma_{d}\left(a_{1} \otimes a_{2} \otimes \ldots \otimes a_{d}\right)=-(-1)^{(d(d-1)) / 2}\left(a_{1} \otimes a_{d} \otimes a_{d-1} \otimes \ldots \otimes a_{3} \otimes a_{2}\right) .
$$

Again note that $\sigma_{d}$ preserves both degree and weight. It is easy to see that the groups of linear transformations of $T^{d}(A)$ generated by $\left\{s_{d}\right\}$ and $\left\{s_{d}, \sigma_{d}\right\}$ are the cyclic group of order $d$ and the dihedral group of order $2 d$, respectively. Let $N_{d}$ and $L_{d}$ be the projections of $T^{d}(A)$ to the invariant subspaces of these two groups. More 
explicitely we have

$$
\begin{aligned}
& N_{d}=(1 / d)\left(1+s_{d}+\ldots+s_{d}^{d-1}\right) \\
& L_{d}=(1 / 2 d)\left(1+s_{d}+\ldots+s_{d}^{d-1}+\sigma_{d}+\sigma_{d} s_{d}+\ldots+\sigma_{d} s_{d}^{d-1}\right) .
\end{aligned}
$$

Proposition 2.5. Let $b$ and $b^{\prime}$ be the boundary maps from $T^{d}(A)$ to $T^{d-1}(A)$ in the Hochschild and Bar complexes for computing $H(A ; A)$ and $H(A ; \mathbb{C})$. Then,

$$
N_{d-1} b=b^{\prime} N_{d} \text { and } L_{d-1} b=b^{\prime} L_{d} .
$$

Definition 2.6. For each $d, w$ let $C_{d, w}(A)$ and $D_{d, w}(A)$ denote the spaces of invariants in $T^{d, w}(A)$ under the groups $\left\langle s_{d}\right\rangle$ and $\left\langle s_{d}, \sigma_{d}\right\rangle$ respectively. Define $\partial_{C}$ : $C_{d, w}(A) \rightarrow C_{d-1, w}(A)$ and $\partial_{D}: D_{d, w}(A) \rightarrow D_{d-1, w}(A)$ by

$$
\begin{aligned}
& \partial_{C}=N_{d-1} b, \\
& \partial_{D}=L_{d-1} b .
\end{aligned}
$$

Proposition 2.7. The maps $\partial_{C}$ and $\partial_{D}$ both square to $0($ on the spaces $C(A)$ and $D(A)$ ). Proof. If $v \in C_{d, w}(A)$ then $v=N_{d} v$. So,

$$
\begin{aligned}
\partial_{C}^{2} v & =\left(N_{d-2} b N_{d-1} b N_{d}\right) v \\
& =\left(N_{d-2} b N_{d-1}{ }^{2} b\right) v \\
& =N_{d-2} b N_{d-1} b^{\prime} v \\
& =N_{d-2}\left(b^{\prime}\right)^{2} v \\
& =0 .
\end{aligned}
$$

Similarly, $\partial_{D}{ }^{2}=0$.

Definition 2.8. Define the bigraded cyclic and dihedral homologies of $A$ by

(Cyclic homology)

$$
H C_{d-1, w}(A)=\left(\left(\operatorname{ker} \partial_{C}\right) \cap C_{d, w}(A)\right) /\left(\left(\operatorname{im} \partial_{C}\right) \cap C_{d, w}(A)\right)
$$

(Dihedral homology)

$$
H D_{d-1, w}(A)=\left(\left(\operatorname{ker} \partial_{D}\right) \cap D_{d, w}(A)\right) /\left(\left(\operatorname{im} \partial_{D}\right) \cap D_{d, w}(A)\right) .
$$

For fixed $w$, the cyclic and dihedral homologies of weight $w$ are defined to be the homologies of the graded complexes,

$$
\begin{aligned}
& \ldots \rightarrow C_{d+1, w}(A) \rightarrow C_{d, w}(A) \rightarrow C_{d-1, w}(A) \rightarrow \ldots \\
& \ldots \rightarrow D_{d+1, w}(A) \rightarrow D_{d, w}(A) \rightarrow D_{d-1, w}(A) \rightarrow \ldots
\end{aligned}
$$

Define the cyclic and dihedral cohomologies of $A$ to be the cohomologies defined by the dual complexes,

$$
\begin{aligned}
& \ldots \rightarrow\left(C_{d-1, w}(A)\right)^{*} \rightarrow\left(C_{d, w}(A)\right)^{*} \rightarrow\left(C_{d+1, w}(A)\right)^{*} \rightarrow \ldots \\
& \ldots \rightarrow\left(D_{d-1, w}(A)\right)^{*} \rightarrow\left(D_{d, w}(A)\right)^{*} \rightarrow\left(D_{d+1, w}(A)\right)^{*} \rightarrow \ldots
\end{aligned}
$$


This notion of cyclic homology was introduced by Connes $[5,6]$ and independently by Tsygan [22]. The definition of cyclic and dihedral homology given here is valid only in characteristic 0 . One can define cyclic and dihedral homology as the homologies of certain double complexes and this definition is the correct one in all characteristics (and of course agrees with the definition given here in characteristic 0 ). For details of this and an excellent exposition of cyclic homology the reader should see Loday and Quillen [16]. For details in the dihedral homology case, one should see Loday [17]. We will need three basic facts concerning cyclic homology, one due to Connes and two due to Loday and Quillen.

Theorem 2.7 (Connes [5]). There is a weight-preserving long exact sequence relating the cyclic homology of $A$ and the Hochschild homology of $A$ :

$$
\ldots \rightarrow H_{d, w}(A ; A) \rightarrow H C_{d, w}(A) \rightarrow H C_{d-2, w}(A) \rightarrow H_{d-1, w}(A ; A) \rightarrow \ldots
$$

Moreover, the map from $H_{d, \mathrm{w}}(A ; A)$ to $H C_{d, \mathrm{w}}(A)$ is the map $N_{d}$.

The other two maps $S: H C_{d, w}(A) \rightarrow H C_{d-2, w}(A)$ and $B: H C_{d-2, w}(A)$ $\rightarrow H_{d-1, w}(A ; A)$ are described in either Connes [5] or Loday and Quillen [16].

Theorem 2.8 (Loday and Quillen [16]). Let $\operatorname{Prim}\left(H L_{d, w}\left(\mathrm{gl}_{n}(\mathbb{C}) \otimes A\right)\right.$ ) denote the primitive elements in the homology of $\mathrm{gl}_{n}(\mathbb{C}) \otimes A$ and let $\operatorname{Prim}\left(H L_{d, w}\left(\mathrm{gl}_{n}(\mathbb{C}) \otimes A\right)\right)^{\mathbf{g}_{n}(\mathbb{C})}$ denote the $\mathrm{gl}_{n}(\mathbb{C})-$ invariants in $\operatorname{Prim}\left(H L_{d, w}\left(\mathrm{gl}_{n}(\mathbb{C}) \otimes A\right)\right)$. Then $\operatorname{dim}\left(\operatorname{Prim}\left(H L_{d, w}\left(\mathrm{gl}_{n}(\mathbb{C}) \otimes A\right)^{g \mathbf{l}_{n}(\mathbb{C})}\right)\right)$ approaches the limit $\operatorname{dim}\left(H C_{d, w}(A)\right)$ as $n \rightarrow \infty$.

More succinctly, $\lim \left(H L\left(\mathrm{gl}_{n}(\mathbb{C}) \otimes A\right)^{\operatorname{gl}_{n}(\mathbb{C})}\right)$ is the graded exterior algebra of $H C(A)$.

The last result we need concerns the case where $A$ is an augmented algebra, i.e., $A=(1 \cdot \mathbb{C}) \oplus I$ where $I$ is an ideal and 1 is a unit.

Theorem 2.9 (Loday and Quillen [16]). Let $A=(1 \cdot \mathbb{C}) \oplus I$ be an augmented algebra. Then $H C_{*, *}(A)=H C_{*, *}(\mathbb{C}) \oplus H C_{*, *}(I)$.

We will need similar results for dihedral homology which are due to Loday. It is easy to verify that $b$ commutes with the maps $\sigma_{d}$. So the Hochschild homology of $A$ splits as a direct sum

$$
H(A ; A)=H(A ; A)^{+} \oplus H(A ; A)^{-}
$$

where $\sigma_{d} v=v$ for $v \in H_{d}(A ; A)^{+}$and $\sigma_{d} v=-v$ for $v \in H_{d}(A ; A)^{-}$.

Proposition 2.10. For the ring $A=A_{+}(k)$ we have

$$
H_{d}\left(A_{+}(k) ; A_{+}(k)\right)=\left\{\begin{array}{lll}
H_{d}\left(A_{+}(k) ; A_{+}(k)\right)^{+} & \text {for } & d \equiv 1,2 \bmod 4 \\
H_{d}\left(A_{+}(k) ; A_{+}(k)\right)^{-} & \text {for } & d \equiv 0,3 \bmod 4
\end{array}\right.
$$

Proof. For any $u \in \operatorname{ker} b$, let $[u]$ denote the homology class in $H\left(A_{+}(k) ; A_{+}(k)\right)$ containing $u$. Recall $x$ and $y$ defined earlier by

$$
\begin{aligned}
& x=1 \otimes t \\
& y=\sum t^{i} \otimes t^{k-i} \otimes t .
\end{aligned}
$$


Clearly $H_{0}\left(A_{+}(k) ; A_{+}(k)\right)$ is in $H\left(A_{+}(k) ; A_{+}(k)\right)^{-}$and $H_{1}\left(A_{+}(k) ; A_{+}(k)\right)$ is in $H\left(A_{+}(k) ; A_{+}(k)\right)^{+}$. We consider $H_{2}\left(A_{+}(k) ; A_{+}(k)\right)$. We begin with a computation.

Claim. For any $a$ with $0 \leqq a \leqq k$, we have

$$
\left[1 \otimes t^{a}\right]=a t^{a-1} \circ[x] .
$$

Proof. The proof is by induction on $a$. For $a=0$, we have $1 \otimes 1=b(1 \otimes 1 \otimes 1)$ so the result holds. For $a>0$,

So,

$$
b\left(1 \otimes t^{a-1} \otimes t\right)=t^{a-1} \otimes t-1 \otimes t^{a}+t \otimes t^{a-1} .
$$

$$
\begin{aligned}
{\left[1 \otimes t^{a}\right] } & =\left[t^{a-1} \otimes t\right]+\left[t \otimes t^{a-1}\right] \\
& =t^{a-1} \circ[x]+t \circ\left((a-1) t^{a-2} \circ[x]\right) \\
& =a t^{a-1} \circ[x], \text { which proves the claim } .
\end{aligned}
$$

Now consider $y-\sigma_{3} y$.

So,

$$
\begin{aligned}
y-\sigma_{3} y & =\sum\left(t^{i} \otimes t^{k-i} \otimes t-t^{i} \otimes t \otimes t^{k-i}\right) \\
& =\sum t^{i} \circ\left(1 \otimes t^{k-i}\right) \circ(1 \otimes t) .
\end{aligned}
$$

$$
\begin{aligned}
{[y]+\sigma_{3}[y] } & =\sum t^{i} \circ\left((k-i) t^{k-i-1}\right) \circ[x] \circ[x] \\
& =0 \text { as }[x] \circ[x]=0 .
\end{aligned}
$$

Next suppose that $A=a_{0} \otimes a_{1} \otimes \ldots \otimes a_{d}$ and $B=b_{0} \otimes a_{d+1} \otimes \ldots \otimes a_{d+e}$ are in $T\left(A_{+}(k)\right)$. Then

$$
\sigma_{d+e+1}(A \circ B)=\sigma_{d+e+1}\left\{\sum \operatorname{sgn}(\tau)\left(a_{0} b_{0}\right) \otimes a_{\tau 1} \otimes \ldots \otimes a_{\tau(d+e)}\right\}
$$

where the sum is over all $\tau$ with $\tau 1<\tau 2<\ldots<\tau d$ and $\tau(d+1)<\ldots<\tau(d+e)$. Note that for such $\tau, \operatorname{sgn}(\tau)$ is the number of pairs $\left(a_{d+i}, a_{j}\right)$ with $1 \leqq j \leqq e$ and $1 \leqq i \leqq d$ such that $a_{d+i}$ occurs to the left of $a_{j}$ in $a_{\tau 1} \otimes \ldots \otimes a_{\tau(d+e)}$.

Applying $\sigma_{d+e+1}$ we have

$$
\sigma_{d+e+1}(A \circ B)=-(-1)^{(d+e)(d+e-1) / 2} \sum \operatorname{sgn}(\tau)\left(a_{0} b_{0}\right) \otimes a_{\tau(d+e)} \otimes \ldots \otimes a_{\tau 1}
$$

where this time the sum is over $\tau$ with $\tau d<\ldots<\tau 1$ and $\tau(d+e)<\ldots<\tau(d+1)$, and $\operatorname{sgn}(\tau)$ is the number of pairs $\left(a_{j}, a_{d+i}\right)$ with $a_{j}$ to the left of $a_{d+i}$ in $a_{\tau(d+e)} \otimes \ldots \otimes a_{\tau 1}$. So

$$
\begin{aligned}
\sigma_{d+e+1}(A \circ B) & = \\
-(-1)^{(d+e)(d+e-1) / 2}\left\{-(-1)^{e(e-1) / 2} \sigma_{e+1}(B)\right\} \circ\left\{-(-1)^{d(d-1) / 2} \sigma_{d+1}(A)\right\} & \\
& =-\sigma_{d+1}(A) \circ \sigma_{e+1}(B)
\end{aligned}
$$

the last equality holding because $(d+e)(d+e-1) / 2=(d(d-1) / 2)+(e(e-1) / 2)$ + de.

Let $[y]^{(r)}$ denote the $r^{\text {th }}$ shuffle power of $[y]$. Since $\sigma_{d+e+1}$ $=-\sigma_{d+1}(A) \circ \sigma_{e+1}(B)$ we have that $t^{i} \circ[y]^{(r)}$ is in $H_{2 r}\left(A_{+}(k) ; A_{+}(k)\right)^{+}$for $r$ odd, and in $H_{2 r}\left(A_{+}(k) ; A_{+}(k)\right)^{-}$for $r$ even. Likewise $t^{i} \circ[y]^{(r)} \circ[x]$ is in 
$H_{2 r+1}\left(A_{+}(k) ; A_{+}(k)\right)^{+}$if $r$ is even and in $H_{2 r+1}\left(A_{+}(k) ; A_{+}(k)\right)^{-}$if $r$ is odd. By Theorem 2.7, the result follows.

We will need two results due to Loday. The first is the analogue of Theorem 2.7 for dihedral homology.

Theorem 2.11 (Loday and Procesi [18]). Let $A$ be a graded $\mathbb{C}$-algebra. Then for each $d$ and $w$, the dimensions of $\operatorname{Prim}\left(H L_{d, w}\left(o_{n}(\mathbb{C}) \otimes A\right)^{o_{n}(\mathbb{C})}\right)$ and $\operatorname{Prim}\left(H L_{d, w}\left(\operatorname{sp}_{2 n}(\mathbb{C}) \otimes A\right)^{\mathbf{s p}_{2 n}(\mathbb{C})}\right)$ approach the same limit as $n$ approaches infinity and this limiting dimension is $\operatorname{dim}\left(H D_{d, w}(A)\right)$. In short, the graded exterior algebra of $H D(A)$ equals $\lim H L\left(o_{n}(\mathbb{C}) \otimes A\right)^{o_{n}(\mathbb{C})}=\lim H L\left(\operatorname{sp}_{2 n}(\mathbb{C}) \otimes A\right)^{\mathrm{sp}_{2 n}(\mathbb{C})}$.

In [17], Loday defines dihedral homology as the homology of a certain double complex. A spectral sequence argument shows that his definition agrees with ours in characteristic 0 . The next result concerns certain homology groups $H T_{*, *}(A)$ which are defined to be the homology of a subcomplex of Loday's double complex for computing dihedral homology. For this application, we won't need the exact definition of $H T(A)$, as so we refer the interested reader to [17] for further details.

Proposition 2.12 (Loday [17]). Let $A$ be a graded $\mathbb{C}$-algebra. Then there exist bigraded homology groups $H T_{*, *}(A)$, and a pair of long exact sequences (2.4) and (2.5) which satisfy:

$$
\begin{aligned}
& \ldots \rightarrow H_{d-1, w}(A ; A)^{-} \rightarrow H_{d, w}(A ; A)^{+} \rightarrow H T_{d, w}(A) \\
& \rightarrow H_{d-2, w}(A ; A)^{-} \rightarrow H_{d-1, w}(A ; A)^{+} \rightarrow \ldots, \\
& \ldots \rightarrow H T_{d, w}(A) \rightarrow H D_{d, w}(A) \rightarrow H D_{d-4, w}(A) \rightarrow H T_{d-1, w}(A) \rightarrow \ldots,
\end{aligned}
$$

In the first sequence (2.4), the map from $H_{d-1, w}(A ; A)^{-}$to $H_{d, w}(A ; A)^{+}$is the map $B$ from Theorem 2.7 .

This completes Sect. 2. In the next section, we will compute the cyclic and dihedral homologies of the truncated polynomial ring $A_{+}(k)$.

\section{Section 3. The cyclic and dihedral homologies of $A_{+}(k)$}

Let $k$ be a positive integer which is fixed for the remainder of this section. Let $A_{+}(k)$ denote the ring $\mathbb{C}[t] /\left(t^{k+1}\right)$, and let $\overline{A_{+}(k)}$ denote $A_{+}(k)$ modulo $\mathbb{C}$. Note that $A_{+}(k)$ is an $\mathbb{N}$-graded ring with the $i^{\text {th }}$ graded piece given by 0 if $i$ exceeds $k$ and by $\mathbb{C} t^{i}$ if $i$ is less than $k+1$. Our aim in this section is to compute the cyclic and dihedral homologies of $A_{+}(k)$ as a graded algebra. As in previous sections, we use $d$ and $w$ for the respective indices of degree and weight and denote the $(d, w)$ bigraded piece of the cyclic homology of $A_{+}(k)$ by $H C_{d, w}\left(A_{+}(k)\right)$. The following theorem is one of the main results of this section.

Theorem 3.1. As a bigraded vector space, the cyclic homology of $A_{+}(k)$ is given by:

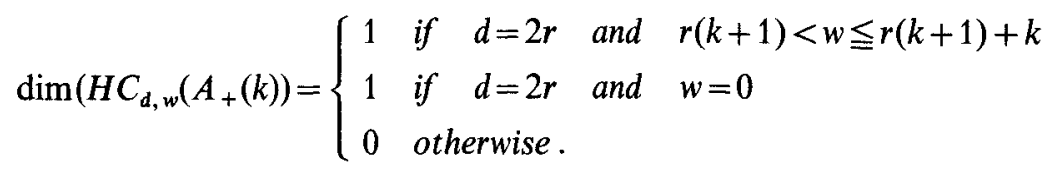


We will actually prove the following proposition which states a slightly stronger result.

Proposition 3.2. Recall the long exact sequence of Connes:

with maps

$$
\ldots \rightarrow H_{d}\left(A_{+}(k) ; A_{+}(k)\right) \rightarrow H C_{d}\left(A_{+}(k)\right) \rightarrow H C_{d-2}\left(A_{+}(k)\right) \rightarrow \ldots
$$

Then,

$$
\begin{aligned}
N: H_{d}\left(A_{+}(k) ; A_{+}(k)\right) & \rightarrow H C_{d}\left(A_{+}(k)\right) \\
S: H C_{d}\left(A_{+}(k)\right) & \rightarrow H C_{d-2}\left(A_{+}(k)\right) \\
B: H C_{d-2}\left(A_{+}(k)\right) & \rightarrow H_{d-1}\left(A_{+}(k) ; A_{+}(k)\right) .
\end{aligned}
$$

(1) $S$ is identically zero on $H C_{* w}\left(A_{+}(k)\right)$ for any nonzero weight $w$.

(2) $S$ is an isomorphism between the 1-dimensional spaces $H C_{2 s, 0}\left(A_{+}(k)\right)$ and $H C_{2 s-2,0}\left(A_{+}(k)\right)$.

(3) For d even, $N$ is an isomorphism of $H_{d}\left(A_{+}(k) ; A_{+}(k)\right)$ onto $H C_{d}\left(A_{+}(k)\right)$. For $d$ odd, $N$ is identically 0.

(4) For $d$ even, $B$ is an isomorphism of $\mathrm{HC}_{d-2}\left(A_{+}(k)\right)$ onto $H_{d-1}\left(A_{+}(k) ; A_{+}(k)\right)$. For $d$ odd, $B$ is identically 0.

(5) $\operatorname{dim}\left(H C_{d}\left(\overline{A_{+}(k)}\right)\right)=\left\{\begin{array}{llll}k & \text { if } d & \text { is even } \\ 0 & \text { is } d & \text { is odd }\end{array}\right.$

Proof. We first prove (1). We will use the following lemma due to Goodwillie ([9], Corollary 11.4.6).

Lemma. Let $D$ be a derivation of $A$ and let $L_{D}$ be the induced homomorphism on cyclic homology. Then $L_{D}{ }^{\circ} S=0$.

We apply this lemma with $A=A_{+}(k)$ and $D$ the derivation $D t^{i}=i t^{i}$. It is easy to see that $L_{D}$ restricted to $H C_{* w}\left(A_{+}(k)\right)$ is just multiplication by $w$. So by the lemma above, $S$ must be identically zero on $H C_{* w}\left(A_{+}(k)\right)$ when $w$ is nonzero. This proves part (1).

We now prove parts (2)-(5) by induction on $d$. Assume that $w$ is nonzero. By (1) we have for each $d$ the short exact sequence

$$
0 \rightarrow H C_{d-1, w}\left(A_{+}(k)\right) \rightarrow H_{d, w}\left(A_{+}(k) ; A_{+}(k)\right) \rightarrow H C_{d, w}\left(A_{+}(k)\right) \rightarrow 0 .
$$

If $d$ is odd then by induction on $d$, the total dimension of $H C_{d-1, *}\left(A_{+}(k)\right)$ in nonzero weight is $k$. By Theorem 2.4, the total dimension of $H_{d, *}\left(A_{+}(k) ; A_{+}(k)\right)$ in nonzero weight is $k$. By the short exact sequence (3.1), the map $B$ is one to one and so $H C_{d, w}\left(A_{+}(k)\right)$ must be zero. This proves statements (3)-(5) for odd values of $d$.

If $d$ is even then $H C_{d-1, w}\left(A_{+}(k)\right)$ is zero for $w$ nonzero by our inductive hypothesis. So by the exact sequence (3.1) the map $N$ is onto for nonzero weight $w$. Also by our induction hypothesis and Theorem 2.4 we have that the total dimensions of $H_{d, *}\left(A_{+}(k) ; A_{+}(k)\right)$ and $H C_{d, *}\left(A_{+}(k)\right)$ in nonzero weight are both $k$. This proves $(3)-(5)$ for nonzero weight.

It remains to prove statement (2) for weight 0 . This follows easily from the observation that $H C_{\mathrm{d}, 0}\left(A_{+}(k)\right)=H C_{d}(\mathbb{C})$. This completes the proof of Proposition 3.2. 
Note that Theorem 3.1 follows from Proposition 3.2 and our previous knowledge of the weights of $H_{d, *}\left(A_{+}(k) ; A_{+}(k)\right)$.

We next compute the dihedral homology of $A_{+}(k)$. We first need the following proposition.

Proposition 3.3. Let $H T\left(A_{+}(k)\right)$ be the homology groups referred to in Proposition 2.12. Then

$$
\operatorname{dim}\left(H T_{d, w}\left(A_{+}(k)\right)\right)=\left\{\begin{array}{l}
\operatorname{dim}\left(H_{d, w}\left(A_{+}(k) ; A_{+}(k)\right)\right) \text { if } \quad d \equiv 2 \bmod 4 \\
\operatorname{dim}\left(H_{d-2, w}\left(A_{+}(k) ; A_{+}(k)\right)\right) \text { if } d \equiv 1 \bmod 4 \\
0 \quad \text { if } \quad d \equiv 0,3 \bmod 4
\end{array}\right.
$$

Proof. We use the long exact sequence (2.4) along with Proposition 2.10. To see how these apply, assume first that $d$ is congruent $2 \bmod 4$. Then we have

$$
H_{d-1}\left(A_{+}(k) ; A_{+}(k)\right)^{-}=H_{d-2}\left(A_{+}(k) ; A_{+}(k)\right)^{+}=0
$$

by Proposition 2.10. So from (2.4) we obtain the exact sequence

$$
\begin{aligned}
0 & \rightarrow H_{d}\left(A_{+}(k) ; A_{+}(k)\right)^{+} \rightarrow H T_{d}\left(A_{+}(k)\right) \rightarrow H_{d-2}\left(A_{+}(k) ; A_{+}(k)\right)^{-} \\
& \rightarrow H_{d-1}\left(A_{+}(k) ; A_{+}(k)\right)^{+} \rightarrow H T_{d-1}\left(A_{+}(k)\right) \rightarrow H_{d-3}\left(A_{+}(k) ; A_{+}(k)\right)^{-} \rightarrow 0 .
\end{aligned}
$$

Also the map between $H_{d-2}\left(A_{+}(k) ; A_{+}(k)\right)^{-}$and $H_{d-1}\left(A_{+}(k) ; A_{+}(k)\right)^{+}$is the map $B$ which is an isomorphism since $d-2$ is even (by Proposition 3.2 (3)). So we have

$$
H T_{d}\left(A_{+}(k)\right) \cong H_{d}\left(A_{+}(k) ; A_{+}(k)\right)^{+} \cong H_{d}\left(A_{+}(k) ; A_{+}(k)\right)
$$

and

$$
H T_{d-1}\left(A_{+}(k)\right) \cong H_{d-3}\left(A_{+}(k) ; A_{+}(k)\right)^{-} \cong H_{d-3}\left(A_{+}(k) ; A_{+}(k)\right) .
$$

If $d \equiv 0 \bmod 4$ then

$$
H_{d}\left(A_{+}(k) ; A_{+}(k)\right)^{+}=H_{d-2}\left(A_{+}(k) ; A_{+}(k)\right)^{-}=0
$$

and

$$
H_{d-1}\left(A_{+}(k) ; A_{+}(k)\right)^{+}=H_{d-3}\left(A_{+}(k) ; A_{+}(k)\right)^{-}=0 .
$$

So from (2.4) we have the exact sequence

$$
0 \rightarrow H T_{d}\left(A_{+}(k)\right) \rightarrow 0 \rightarrow 0 \rightarrow H_{d-1}\left(A_{+}(k)\right) \rightarrow 0
$$

which completes the proof.

Theorem 3.4. The dihedral homology of $A_{+}(k)$ is given by

$\operatorname{dim}\left(H D_{d, w}\left(A_{+}(k)\right)\right)=\left\{\begin{array}{l}1 \text { if } d=(4 s)-2 \text { and } w=(2 s-1)(k+1)+t \text { for } 1 \leqq t \leqq k \\ 0 \text { otherwise. }\end{array}\right.$

Moreover, for those $d, w$ with $H D_{d, w}\left(A_{+}(k)\right)$ nonzero, the map $L_{d}$ from $H_{d, w}\left(A_{+}(k) ; A_{+}(k)\right)$ into $H D_{d, w}\left(A_{+}(k)\right)$ is an isomorphism. 
Proof. The proof is structured very much like the proof of Proposition 3.2. We proceed by induction on $d$ and use the long exact sequence (2.5) which relates dihedral homology to the homology groups $H T\left(A_{+}(k)\right)$.

To begin our induction, we have from (2.5) the exact sequences

$$
0 \rightarrow H T_{i}\left(A_{+}(k)\right) \rightarrow H D_{i}\left(A_{+}(k)\right) \rightarrow 0
$$

for $0 \leqq i \leqq 3$. The map from $H T_{i}\left(A_{+}(k)\right)$ to $H D_{i}\left(A_{+}(k)\right)$ is the inclusion map. By Proposition 3.3 we have

$$
H T_{i, w}\left(A_{+}(k)\right)=\left\{\begin{array}{l}
0 \text { for } i=0,1,3 \\
H_{2}\left(A_{+}(k) ; A_{+}(k)\right) \text { for } i=2
\end{array}\right.
$$

with equality in the case $i=2$ achieved by the inclusion of $H_{2}\left(A_{+}(k) ; A_{+}(k)\right)$ in $H T_{2}\left(A_{+}(k)\right)$.

Assume the result is known for $d<4 s$. By our induction hypotheses,

$$
H D_{4 s-4}\left(A_{+}(k)\right)=H D_{4 s-3}\left(A_{+}(k)\right)=H D_{4 s-1}\left(A_{+}(k)\right)=0 .
$$

Also by Proposition 3.3 we have

$$
H T_{4 s+3}\left(A_{+}(k)\right)=H T_{4 s}\left(A_{+}(k)\right)=0 .
$$

Using these facts in the sequence (2.5) we obtain exact sequences,

$$
\begin{gathered}
0 \rightarrow H D_{4 s+3, w}\left(A_{+}(k)\right) \rightarrow 0 \\
0 \rightarrow H_{4 s+2, w}\left(A_{+}(k) ; A_{+}(k)\right) \rightarrow H D_{4 s+2, w}\left(A_{+}(k)\right) \\
\rightarrow H D_{4 s-2, w}\left(A_{+}(k)\right) \rightarrow H_{4 s-1, w}\left(A_{+}(k) ; A_{+}(k)\right) \rightarrow H D_{4 s+1, w}\left(A_{+}(k)\right) \rightarrow 0 . \\
0 \rightarrow H D_{4 s, w}\left(A_{+}(k)\right) \rightarrow 0 .
\end{gathered}
$$

The sequences (3.2) and (3.4) immediately imply that

$$
H D_{4 s, *}\left(A_{+}(k)\right)=H D_{4 s+3, *}\left(A_{+}(k)\right)=0 .
$$

In (3.3) the map from $H_{4 s+2, w}\left(A_{+}(k) ; A_{+}(k)\right)$ into $H D_{4 s+2, w}\left(A_{+}(k)\right)$ is inclusion (equivalently the map $L_{4 s+2}$ ). Also, by our induction hypotheses,

$$
\operatorname{dim}\left(H D_{4 s-2, w}\left(A_{+}(k)\right)\right)=\operatorname{dim}\left(H_{4 s-1, w}\left(A_{+}(k) ; A_{+}(k)\right) .\right.
$$

So to complete our induction step, it remains to show that $H D_{4 s+1, w}\left(A_{+}(k)\right)=0$.

To show that $H D_{4 s+1, w}\left(A_{+}(k)\right)$ is zero, we use the same argument as in the proof of Proposition 3.2. All that is required is a generalization of Goodwillie's Lemma which is applicable in this case. This generalization follows easily from the version stated earlier. We leave the details to the reader.

The next result summarizes the two main results of this section in terms of cyclic and dihedral cohomology. Although these results follow immediately from their homological versions, we state them here because we will actually work with cyclic and dihedral cohomology in later sections. 
Corollary 3.5. The cyclic and dihedral cohomologies of $A_{+}(k)$ are given by:

$\operatorname{dim}\left(H C^{d, w}\left(A_{+}(k)\right)\right)= \begin{cases}1 & \text { if } d=2 s \text { and } w=((k+1) s)+t \text { with } 1 \leqq t \leqq k \\ 0 & \text { otherwise, }\end{cases}$ $\operatorname{dim}\left(H D^{d, w}\left(A_{+}(k)\right)\right)= \begin{cases}1 & \text { if } d=4 s+2, w=((k+1)(2 s+1))+t \text { with } 1 \leqq t \leqq k \\ 0 & \text { otherwise }\end{cases}$

To end this section we display explicit representatives for the cyclic cohomology classes of $A_{+}(k)$. First choose a basis for $C\left(A_{+}(k)\right)$ in which each element is of the form $N_{d}\left(t^{u_{1}} \otimes \ldots \otimes t^{u_{d}}\right)$. For $d$ odd, there is a unique such basis and for $d$ even, the basis elements are unique up to sign. Let $\langle$,$\rangle be the bilinear form whose$ matrix with respect to this chosen basis is the identity. Using the nondegenerate bilinear form $\langle$,$\rangle , identify C\left(A_{+}(k)\right)^{*}$ with $C\left(A_{+}(k)\right)$ and thus view $\delta_{C}=\partial_{C}{ }^{t}$ as a map from $C_{d, w}\left(A_{+}(k)\right)$ to $C_{d+1, w}\left(A_{+}(k)\right)$. It is easy to check that with this identification $\delta_{C}$ is given by,

$$
\delta_{C}\left(t^{u_{1}} \ldots t^{u_{d}}\right)=\sum(-1)^{i-1} t^{u_{1}} \ldots t^{u_{i-1}}\left\{\sum t^{j} t^{u_{i}-j}\right\} \ldots t^{u_{d}}
$$

where the first sum is on $i$ from 1 to $d$ and the second sum is on $j$ from 1 to $u_{i}-1$. We will use the following piece of notation. For $u$ a positive integer define $d_{k}\left(t^{k+u}\right)$ to be

$$
d_{k}\left(t^{k+u}\right)=\left\{\sum t^{l} \otimes t^{k+u-l}\right\}-\left\{\sum\left(t^{l} \otimes t^{k+u-l}\right)+\left(t^{k+u-l} \otimes t^{l}\right)\right\}
$$

Here the first sum is on $l$ from 1 to $k+u-1$ and the second sum is on $l$ from 1 to $u-1$.

Let $\beta$ be the dual of the boundary map $b^{\prime}$ from the Bar complex for $A_{+}(k+u)$. Then $d_{k}\left(t^{k+u}\right)$ is $\beta\left(t^{k+u}\right)$ minus any terms which involve a higher power of $t$ than $k$. In particular, $d_{k}\left(t^{k+u}\right)$ consists of terms involving only $t, t^{2}, \ldots, t^{k}$. Note also that

$$
\beta\left(d_{k}\left(t^{k+u}\right)\right)=-\sum d_{k}\left(t^{k+l}\right) \otimes t^{u-l}+\sum t^{l} \otimes d_{k}\left(t^{k+u-l}\right) .
$$

Definition 3.6. For $s$ a non-negative integer and $1 \leqq N \leqq k$, define $\Delta_{N, s}$ to be

$$
\Delta_{N, s}=N_{2 s+1}\left\{\sum \sum i t^{i} \otimes d_{k}\left(t^{k+1+u_{1}}\right) \otimes d_{k}\left(t^{k+1+u_{2}}\right) \otimes \ldots \otimes d_{k}\left(t^{k+1+u_{s}}\right)\right\},
$$

where the first sum is on $i$ from 1 to $N$ and the second sum is over all ordered sequences $\left(u_{1}, \ldots, u_{s}\right)$ of non-negative integers which sum to $N-i$.

Note that $\Delta_{N, s}$ is in $C_{2 s+1,(k+1) s+N}\left(A_{+}(k)\right)$. We will show that $\Delta_{N, s}$ is a cyclic cohomology representative for the nonzero class of degree $2 s+1$ and weight $(k+1) s+N$.

Lemma 3.7. $\delta_{C}\left(\Delta_{N, s}\right)=0$.

Proof. We separate $\delta_{C}\left(\Delta_{N, s}\right)$ into several parts.

$$
\delta_{C}\left(\Delta_{N, \mathrm{~s}}\right)=A+B+C+D+E,
$$


where

$$
\begin{gathered}
A=\sum \sum \sum i t^{j} t^{i-j} d_{k}\left(t^{k+1+u_{1}}\right) \ldots d_{k}\left(t^{k+1+u_{s}}\right) \\
B=-\sum \sum \sum i t^{i} t^{l} d_{k}\left(t^{k+1+u_{1}-l}\right) d_{k}\left(t^{k+1+u_{2}}\right) \ldots d_{k}\left(t^{k+1+u_{s}}\right) \\
C=\sum \sum \sum \sum i t^{i} d_{k}\left(t^{k+1+u_{1}}\right) \ldots\left(d_{k}\left(t^{k+1+u_{j}-l}\right) t^{l}\right) \ldots d_{k}\left(t^{k+1+u_{s}}\right) \\
D=-\sum \sum \sum \sum i t^{i} d_{k}\left(t^{k+1+u_{1}}\right) \ldots\left(t^{l} d_{k}\left(t^{k+1+u_{j}-l}\right)\right) \ldots d_{k}\left(t^{k+1+u_{s}}\right),
\end{gathered}
$$

and

$$
E=\sum \sum \sum i t^{i} d_{k}\left(t^{k+1+u_{1}}\right) \ldots d_{k}\left(t^{k+1+u_{s}-l}\right) t^{l} .
$$

Note that $C+D=0$ as the term in $C$ corresponding to the summation indices $i$, $\left(u_{1}, \ldots, u_{s}\right), j, l$ can be thought of as cancelling the term in $D$ corresponding to the indices $i,\left(u_{1}, \ldots, u_{j}-l, u_{j+1}+l, \ldots, u_{s}\right), j+1, l$.

Also $A$ can be rewritten as

$$
A=\sum \sum(x+y) t^{x} t^{y} d_{k}\left(t^{k+1+u_{1}}\right) \ldots d_{k}\left(t^{k+1+u_{s}}\right),
$$

where the first sum is over all pairs $x, y$ with $x+y \leqq N$ and the second sum is over all $\left(u_{1}, \ldots, u_{s}\right)$ with $u_{i} \geqq 0$ and $\sum u_{i}=N-(x+y)$. This in turn can be rewritten as $A=A_{1}+A_{2}$ where

$$
A_{1}=\sum \sum x t^{x} t^{y} d_{k}\left(t^{k+1+u_{1}}\right) \ldots d_{k}\left(t^{k+1+u_{s}}\right)
$$

and

$$
A_{2}=-\sum \sum y t^{y} d_{k}\left(t^{k+1+u_{1}}\right) \ldots d_{k}\left(t^{k+1+u_{s}}\right) t^{x} .
$$

Clearly $A_{1}=-B$ and $A_{2}=-E$ which proves the lemma.

Proposition 3.8. $\Delta_{N, s}$ is a representative for the nonzero cyclic cohomology class of $A_{+}(k)$ of degree $2 s+1$ and weight $(k+1) s+N$.

Proof. Fix $s$ non-negative and $N$ in the range 1 to $k$. Let $W, V$, and $U$ be the spaces $C_{2 s+i,(k+1) s+N}\left(A_{+}(k)\right)$ for $i=0,1$, and 2 . Let $D$ and $E$ be the matrices for $\partial_{c}: U \rightarrow V$ and $\partial_{C}: V \rightarrow W$ respectively. Here the matrices are taken with respect to the bases for $U, V, W$ described earlier. Let $R$ denote the row space of $E$ and let $C$ denote the column space of $D$.

We know that $C C R^{\perp}$ and that $\operatorname{dim}\left(R^{\perp} / C\right)=1$. By Proposition 3.2, we also know that

$$
R^{\perp}=C \oplus \mathbb{C}_{N, s} .
$$

A vector $v$ is a cohomology representative if and only is $v \in \operatorname{ker}\left(D^{t}\right)$ and $v \in \operatorname{im}\left(E^{t}\right)$. These latter two conditions are equivalent to $v \in C^{\perp}$ and $v \in R$. The previous lemma shows that $\Delta_{N, s} \in C^{\perp}$. To show that $\Delta_{N, s} \in R$ it suffices (by (3.5)) to show that $\left\langle\Delta_{N, s}, \eta_{N, s}\right\rangle$ is nonzero. The key observation is that in terms of our chosen basis $\left\{b_{i}\right\}$ for $C_{2 s+1,(k+1) s+N}\left(A_{+}(k)\right)$, both $\Delta_{N, s}$ and $\eta_{N, s}$ have non-negative coefficients. To be precise,

$$
A_{N, s}=\sum p_{i} b_{i} \text { and } \eta_{N, s}=\sum q_{i} b_{i},
$$


where the $p_{i}$ and the $q_{i}$ are non-negative integers. Hence the inner product of $\Delta_{N, s}$ and $\eta_{N, s}$ is nonzero provided there exists an $i$ with $p_{i}$ and $q_{i}$ both nonzero. Such a $b_{i}$ is

$$
b_{i}=N_{d}\left(t^{N} \otimes t^{k} \otimes t \otimes \ldots \otimes t^{k} \otimes t\right) .
$$

This completes the proof of Proposition 3.8.

\section{Section 4. Projection to finite $n$}

Part I-Cyclic cohomology

Let $z_{i, j}$ for $i, j \in\{1,2, \ldots, n\}$ be the matrix with a 1 in the $i, j$ entry and 0 's elsewhere, and let $A$ be an $\mathbb{N}$-graded $\mathbb{C}$-algebra with homogeneous basis $\mathfrak{B}=\left\{b_{u}: u=1,2, \ldots\right\}$. The set $\left\{z_{i, j} \otimes b_{u}\right\}$ is a basis for the Lie algebra $L=\mathrm{gl}_{n}(\mathbb{C}) \otimes A$. Let $\left\{\left(z_{i, j} \otimes b_{u}\right)^{*}\right\}$ denote the dual basis of $L^{*}$.

Recall the boundary map $\partial: \wedge^{r} L \rightarrow \wedge^{r-1} L$ in the Koszul complex for computing $H L_{*}\left(\mathrm{gl}_{n}(\mathbb{C}) \otimes A\right)$,

$$
\partial\left(\alpha_{1} \wedge \ldots \wedge \alpha_{r}\right)=\sum(-1)^{i+j}\left[\alpha_{i}, \alpha_{j}\right] \wedge \alpha_{1} \wedge \ldots \wedge \hat{\alpha}_{i} \wedge \ldots \wedge \hat{\alpha}_{j} \wedge \ldots \wedge \alpha_{r} .
$$

We will need an explicit formula for the dual of $\partial$, which we denote by $\delta$. For the purpose of this formula, let $d$ be the map from $A \otimes A$ to $A$ given by

$$
d(a \otimes b)=a b .
$$

Let $d^{*}$ be the dual map from $A^{*}$ to $A^{*} \otimes A^{*}$, written explicitely as

$$
d^{*}\left(b_{u}{ }^{*}\right)=\sum c_{u, v, w} b_{v}{ }^{*} \otimes b_{w}{ }^{*}
$$

(since $A$ is $\mathbb{N}$-graded, hence finite dimensional in each graded piece, the above sum is finite). The next lemma is a simple computation. The proof is left to the reader.

Lemma 4.1. In terms of the natural basis of $\wedge\left(L^{*}\right)$ extending the basis $\left\{\left(z_{i, j} \otimes b_{u}\right)^{*}\right\}$ of $L^{*}$, the map $\delta$ is given by

$$
\begin{aligned}
\delta\left(\left(z_{i_{1}, j_{1}} \otimes b_{u_{1}}\right)^{*} \wedge\left(z_{i_{2}, j_{2}} \otimes b_{u_{2}}\right)^{*} \wedge \ldots \wedge\left(z_{i_{d}, j_{d}} \otimes b_{u_{d}}\right)^{*}\right) & \\
= & \sum\left(z_{i_{1}, j_{1}} \otimes b_{u_{1}}\right)^{*} \wedge \ldots \wedge\left\{(-1)^{s-1} \sum \sum c_{u_{s}, v, w}\left(z_{i_{s}, l} \otimes b_{v}\right)^{*} \wedge\left(z_{l, j_{s}} \otimes b_{w}\right)^{*}\right\} \\
& \wedge \ldots \wedge\left(z_{i_{d}, j_{d}} \otimes b_{u_{d}}\right)^{*} .
\end{aligned}
$$

In the case that $A=A_{+}(k)$ with basis $1, t, \ldots, t^{k}$ the result of Lemma 4.1 is formula (4.1) below:

(4.1) For $L=\mathrm{gl}_{n}(\mathbb{C}) \otimes A_{+}(k)$ with basis $\left\{z_{i, j} \otimes t^{u}\right\}$ we have

$$
\begin{aligned}
\delta\left(\left(z_{i_{1}, j_{1}} \otimes t^{u_{1}}\right)^{*} \wedge \ldots \wedge\left(z_{i_{d}, j_{d}} \otimes t^{u_{d}}\right)^{*}\right) & \\
= & \sum\left(z_{i_{1}, j_{1}} \otimes t^{u_{1}}\right)^{*} \wedge \ldots \wedge\left\{(-1)^{s-1} \sum \sum\left(z_{i_{s}, l} \otimes t^{\nu}\right)^{*} \wedge\left(z_{l, j_{s}} \otimes t^{u_{s}-v}\right)^{*}\right\} \\
& \wedge \ldots \wedge\left(z_{i_{d}, i_{d}} \otimes t^{u_{d}}\right)^{*} .
\end{aligned}
$$

Recall the complex $\left(C^{*, *}(A), \delta_{C}\right)$ for computing the cyclic cohomology of $A$. The vector space $C^{d, *}(A)$ is the dual space of $C_{d, *}(A)$ where $C_{d-1, *}(A)$ is $T^{d}(A)$ 
modulo the relations

$$
\alpha_{1} \otimes \ldots \otimes \alpha_{d}=(-1)^{d-1} \alpha_{2} \otimes \ldots \otimes \alpha_{d} \otimes \alpha_{1} .
$$

The coboundary $\delta_{C}$ is given explicitely in terms of the basis $\mathfrak{B}$ by

$$
\delta_{C}\left(b_{u_{1}}{ }^{*} \otimes \ldots \otimes b_{u_{d}}{ }^{*}\right)=\sum b_{u_{1}}{ }^{*} \otimes \ldots \otimes\left\{(-1)^{s-1} \sum c_{u_{s}, v, w} b_{v}{ }^{*} \otimes b_{w}{ }^{*}\right\} \otimes \ldots \otimes b_{u_{d}}{ }^{*} .
$$

Define the ring $R C(A)$ by saying that $R C(A)$ is the tensor algebra of $C(A)$ modulo the relations

$$
\alpha \otimes \beta=(-1)^{(r+1)(s+1)} \beta \otimes \alpha \quad \text { for } \quad \alpha \in C^{r, *}(A), \quad \beta \in C^{s, *}(A) .
$$

For $\alpha_{i} \in C^{d_{i}, w_{i}}(A)(i=1,2, \ldots, p)$ let $\alpha_{1} \cdot \alpha_{2} \cdot \ldots \cdot \alpha_{p}$ denote the image of $\alpha_{1} \otimes \ldots \otimes \alpha_{p}$ in $R C(A)$. Define the degree and weight of $\alpha_{1} \cdot \ldots \cdot \alpha_{p}$ by

$$
\begin{aligned}
\operatorname{deg}\left(\alpha_{1} \cdot \ldots \cdot \alpha_{p}\right) & =\left(d_{1}+1\right)+\left(d_{2}+1\right)+\ldots+\left(d_{p}+1\right) \\
w t\left(\alpha_{1} \cdot \ldots \cdot \alpha_{p}\right) & =w_{1}+\ldots+w_{p} .
\end{aligned}
$$

Bigrade $R C(A)$ by degree and weight and let $R C^{d, w}(A)$ denote its $(d, w)$-graded piece.

Extend $\delta_{C}$ to an antiderivation on $R C(A)$. Thus

$$
\delta_{C}(\alpha \cdot \beta)=\left(\delta_{c}(\alpha)\right) \cdot \beta+(-1)^{r-1} \alpha \cdot\left(\delta_{C}(\beta)\right)
$$

for $\alpha \in R C^{r, *}(A)$. Define $H R C^{*, *}(A)$ to be the bigraded cohomology of the complex $\left(R C(A), \delta_{C}\right)$.

The next result is well-known.

Proposition 4.2. Let $A$ be a graded $\mathbb{C}$-algebra. As a ring, $H R C(A)$ is isomorphic to the tensor product of the exterior algebra of the even degree part of $H C(A)$ and the symmetric algebra of the odd degree part of $H C(A)$. In short,

$$
H R C(A)=\wedge\left(H C^{\text {even }}(A)\right) \otimes \operatorname{Sym}\left(H C^{\text {odd }}(A)\right) .
$$

Definition 4.3. Define $\Phi_{n}: C(A) \rightarrow \wedge\left(\mathrm{gl}_{n}(\mathbb{C}) \otimes A\right)$ by

$$
\Phi_{n}\left(N_{d}\left(a_{1}^{*} \otimes \ldots \otimes a_{d}^{*}\right)\right)=\sum\left(z_{i_{1}, i_{2}} \otimes a_{1}\right)^{*} \otimes\left(z_{i_{2}, i_{3}} \otimes a_{2}\right)^{*} \otimes \ldots \otimes\left(z_{i_{d}, i_{1}} \otimes a_{d}\right)^{*},
$$

where the sum is over all $i_{1}, \ldots, i_{d}$ with $1 \leqq i_{j} \leqq n$.

It is easy to check that the sum on the right is annihilated by $\mathrm{gl}_{n}(\mathbb{C})$ and is mapped to $(-1)^{d-1}$ times itself when the $a_{i}$ are permuted cyclically. Thus $\Phi_{n}$ is a well-defined weight preserving map from $C(A)$ to $\left(\wedge\left(\mathrm{gl}_{n}(\mathbb{C}) \otimes A\right)^{\mathrm{g} \ln _{n}(\mathbb{C})}\right.$ which raises degree by 1 . If $\alpha \in C^{r, *}(A)$ and $\beta \in C^{s, *}(A)$ then

$$
\Phi_{n}(\alpha) \wedge \Phi_{n}(\beta)=(-1)^{(r+1)(s+1)} \Phi_{n}(\beta) \wedge \Phi_{n}(\alpha) .
$$

Thus $\Phi_{n}$ extends to a degree and weight preserving homomorphism of the ring $R C(A)$ into the ring $\wedge\left(\mathrm{gl}_{n}(\mathbb{C}) \otimes A\right)^{\mathbf{g l}_{n}(\mathbb{C})}$. 
Comparing Lemma 4.1 with formula (4.3) we have

$$
\delta\left(\Phi_{n}\left(N_{d}\left(a_{1} \otimes \ldots \otimes a_{d}\right)\right)\right)=\Phi_{n}\left(\delta_{c}\left(N_{d}\left(a_{1} \otimes \ldots \otimes a_{d}\right)\right)\right)
$$

for all $a_{1}, \ldots, a_{d} \in A^{*}$. Thus $\Phi_{n}$ gives a ring homomorphism (also called $\Phi_{n}$ ) from $H R C(A)$ to $H L\left(\mathrm{gl}_{n}(\mathbb{C}) \otimes A\right)^{g 1_{n}(\mathbb{C})}$. The next result is implicit in the work of Quillen and Loday but can also be found in Tsygan [22].

Theorem 4.4. Let $A$ be a graded $\mathbb{C}$-algebra. Then for $d<n$ and for all $w, \Phi_{n}$ is an isomorphism of $H R C^{d, w}(A)$ onto $H L^{d, w}\left(\mathrm{gl}_{n}(\mathbb{C}) \otimes A\right)^{\mathrm{g}_{n}(\mathbb{C})}$.

Our goal in the rest of this section is to obtain more information about the image of map $\Phi_{n}$ in degrees $d>n$. Note that $C(\mathbb{C})$ has dimension 1 at each even degree and dimension 0 at each odd degree. So $H R C(\mathbb{C})$ is an exterior algebra with one generator $R(2 s-1)=1 \otimes 1 \otimes \ldots \otimes 1$ in each odd degree $2 s-1$. The next proposition can either be proved by topological means or can be obtained as a direct consequence of Theorem 5.4 in Kostant [14].

Proposition 4.5. Let $A=\mathbb{C}$ and let $G(n)$ and $F(n)$ be the subalgebras of $H R C(\mathbb{C})$ generated by $\{R(s): s \leqq 2 n\}$ and $\{R(s): s>2 n\}$ respectively. Then $\Phi_{n}(F(n))=0$ and $\Phi_{n}$ restricted to $G(n)$ is an isomorphism of $H R C(\mathbb{C})$ onto $H L\left(\mathrm{gl}_{n}(\mathbb{C})\right.$ ).

Let $A(k)$ denote the ring $\mathbb{C}[t] /\left(1-t^{k+1}\right)$. The next lemma determines the image of $\Phi_{n}$ in the case $A=A(k)$.

Lemma 4.6. Let $\mathscr{G}$ be a semisimple complex Lie algebra, and let $H L_{*}(\mathscr{G} \otimes A(k))^{\mathscr{G}}$ denote the $\mathscr{G}$-invariants in the Lie algebra homology of $\mathscr{G} \otimes A(k)$. Then $H L_{*}(\mathscr{G} \otimes A(k))^{\mathscr{g}}$ is isomorphic to the tensor product of $k+1$ copies of $H L_{*}(\mathscr{G})$. In particular,

$\operatorname{dim}\left(\operatorname{Prim}\left(H L_{d}(\mathscr{G} \otimes A(k))^{\mathscr{G}}\right)\right)=\left\{\begin{array}{l}k+1 \text { if } d=2 e+1 \text { for } e \text { an exponent of } \mathscr{G} \\ 0 \text { otherwise }\end{array}\right.$

Proof. Let $\omega=e^{2 \pi i /(k+1)}$. For $j \in\{0,1, \ldots, k\}$ and $x \in \mathscr{G}$, let $x_{j}$ be defined by

Let $\mathfrak{M}_{j}=\left\{x_{j}: x \in \mathscr{G}\right\}$.

$$
x_{j}=\sum \omega^{j s}\left(x \otimes t^{s}\right) .
$$

It is easy to check that $\left[x_{j}, y_{m}\right]=\delta_{j, m}[x, y]_{j}$. Thus

$$
\mathscr{G} \otimes A(k)=\mathfrak{M}_{0} \oplus \mathfrak{M}_{1} \oplus \ldots \oplus \mathfrak{M}_{k}
$$

is a Lie algebra direct sum, and so $\mathscr{G} \otimes A(k)$ is isomorphic to a direct sum of $k+1$ copies of $\mathscr{G}$. The result follows.

Recall that $C_{d, w}\left(A_{+}(k)\right)$ denotes the complex for computing the cyclic homology of $A_{+}(k)$. In what follows we use the notation $\beta(z)$ for $z$ an integer to mean $z-(k+1)$ if $z>k$ and 0 otherwise.

Definition 4.7. Define $\partial_{C}{ }^{-}: C_{d, w}\left(A_{+}(k)\right) \rightarrow C_{d-1, w-k-1}\left(A_{+}(k)\right)$ by

$$
\begin{aligned}
\partial_{C}{ }^{-} & \left(N_{d}\left(t^{u_{1}} \otimes \ldots \otimes t^{u_{d}}\right)\right) \\
= & N_{d-1}\left\{\sum(-1)^{i-1} \beta\left(u_{i}+u_{i+1}\right) t^{u_{1}} \otimes \ldots \otimes t^{u_{i}+u_{i+1}-(k+1)} \otimes \ldots \otimes t^{u_{d}}\right. \\
& \left.+(-1)^{d-1} \beta\left(u_{d}+u_{1}\right) t^{u_{1}+u_{d}-(k+1)} \otimes t^{u_{2}} \otimes \ldots \otimes t^{u_{d-1}}\right\} .
\end{aligned}
$$


The following facts are easily verified.

(1) $\left(\partial_{c}{ }^{-}\right)^{2}=0$,

(2) $\partial_{c}{ }^{+} \partial_{C}{ }^{-}=-\partial_{c}{ }^{-} \partial_{C}{ }^{+}$,

(3) $\partial_{C}{ }^{-}$lowers degree by 1 and weight by $(k+1)$.

So $\left(C_{d, w}\left(A_{+}(k)\right), \partial_{c}{ }^{+}, \partial_{c}{ }^{-}\right)$constitutes a double complex with the slightly unusual bigrading indicated in the picture below:

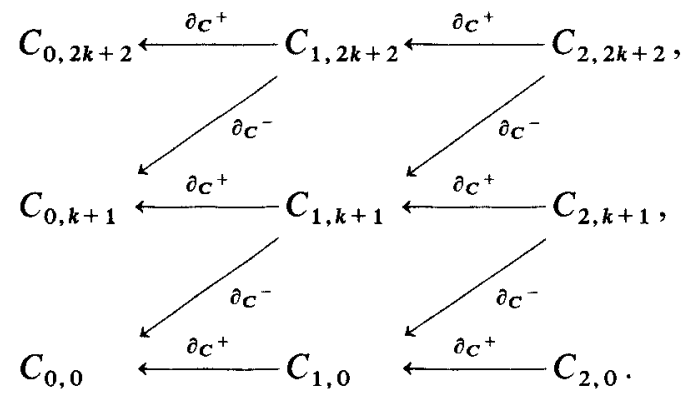

Let $\operatorname{Tot}\left(C\left(A_{+}(k)\right)\right)$ be the associated total complex and let $H^{\text {tot }}{ }_{d}\left(A_{+}(k)\right)$ be the homology of this total complex. We will need the following result.

Theorem 4.8. Let $A(k)$ denote the ring $\mathbb{C}[t] /\left(1-t^{k+1}\right)$. Then $H^{\text {tot }}{ }_{*}\left(A_{+}(k)\right)$ $=H C_{*}(A(k))$. Moreover,

$$
\operatorname{dim}\left(H C_{d}(A(k))\right)=\left\{\begin{array}{l}
k+1 \text { if } d \text { is even } \\
0 \text { if } d \text { is odd } .
\end{array}\right.
$$

Proof. Note that as vector spaces $A_{+}(k)$ and $A(k)$ are both isomorphic to $\mathbb{C} \oplus \mathbb{C} t \oplus \mathbb{C} t^{2} \oplus \ldots \oplus \mathbb{C} t^{k}$. Thus the usual complexes $C_{d}\left(A_{+}(k)\right)$ and $C_{d}(A(k))$ for their cyclic homologies are identical as vector spaces.

Let $t^{u_{1}} \otimes t^{u_{2}} \otimes \ldots \otimes t^{u_{d}}$ be a basis vector for $C(A(k))$ and let $\partial_{c}$ be the differential in $C(A(k))$. Then

$$
\begin{aligned}
\partial_{C}( & \left.N_{d}\left\{t^{u_{1}} \otimes t^{u_{2}} \otimes \ldots \otimes t^{u_{d}}\right\}\right) \\
= & N_{d-1}\left\{\Sigma(-1)^{i-1}\left(1-\beta\left(u_{i}+u_{i+1}\right)\right) t^{u_{1}} \otimes \ldots \otimes t^{u_{i}+u_{i+1}} \otimes \ldots \otimes t^{u_{d}}\right. \\
& \left.+\beta\left(u_{i}+u_{i+1}\right) t^{u_{1}} \otimes \ldots \otimes t^{u_{i}+u_{i+1}-(k+1)} \otimes \ldots \otimes t^{u_{d}}\right\} \\
& +(-1)^{d-1} N_{d-1}\left\{\left(1-\beta\left(u_{d}+u_{1}\right)\right) t^{u_{1}+u_{d}} \otimes t^{u_{2}} \otimes \ldots \otimes t^{u_{d-1}}\right. \\
& \left.+\beta\left(u_{d}+u_{1}\right) t^{u_{1}+u_{d}-(k+1)} \otimes t \otimes \ldots \otimes t^{u_{d}-1}\right\} .
\end{aligned}
$$

Observe that

$$
\begin{aligned}
\partial_{C}{ }^{+} & \left(t^{u_{1}} \otimes \ldots \otimes t^{u_{d}}\right) \\
= & N_{d-1}\left\{\sum(-1)^{i-1}\left(1-\beta\left(u_{i}+u_{i+1}\right)\right) t^{u_{1}} \otimes \ldots \otimes t^{u_{i}+u_{i+1}} \otimes \ldots \otimes t^{u_{d}}\right. \\
& \left.+(-1)^{d-1}\left(1-\beta\left(u_{1}+u_{d}\right)\right) t^{u_{1}+u_{d}} \otimes t^{u_{2}} \otimes \ldots \otimes t^{u_{d-1}}\right\} .
\end{aligned}
$$

From this it follows that $H_{d}^{\text {tot }}\left(A_{+}(k)\right)=H C_{d}(A(k))$.

By the result of Loday and Quillen, Theorem 2.8, we have

$$
H C_{*}(A(k))=\lim \operatorname{Prim}\left(H_{*}\left(\mathrm{gl}_{n}(\mathbb{C}) \otimes A(k)\right)^{\mathbf{g l}_{n}(\mathbb{C})}\right) .
$$


By Lemma 4.6, the primitive part of $H_{d}\left(\mathrm{gl}_{n}(\mathbb{C}) \otimes A(k)\right)^{\mathrm{gl}_{n}(\mathbb{C})}$ has dimension $k+1$ if $d$ is $2 e+1$ for $e$ an exponent of $\mathrm{gl}_{n}(\mathbb{C})$ and 0 otherwise. Since the exponents of $\mathrm{gl}_{n}(\mathbb{C})$ are $0,1,2, \ldots, n-1$. Theorem 4.8 follows.

We are now ready to state, for $\mathrm{gl}_{n}(\mathbb{C})$, one of the results alluded to in Sect. 1.

Theorem 4.9. Let $D(n)$ and $E(n)$ be the exterior algebras generated by the generators of $H R C^{*, *}\left(A_{+}(k)\right)$ of degrees greater than $2 n$ and less than $2 n$ respectively. Then

1) $\Phi_{n}$ maps $E(n)$ isomorphically into $H L\left(\mathrm{~g} 1_{n}(\mathbb{C}) \otimes A_{+}(k)\right)$.

2) If the map $\Phi_{n}: H R C\left(A_{+}(k)\right) \rightarrow H L\left(\mathrm{gl}_{n}(\mathbb{C}) \otimes A_{+}(k)\right)$ is onto then $\Phi_{n}\left(H R C\left(A_{+}(k)\right)\right)=\Phi_{n}(E(n))$.

Proof. We first prove 1).

For each $d \in\{1,3, \ldots, 2 n-1\}$ and each $w$ of the form $((k+1)(d-1) / 2)+t$ $(1 \leqq t \leqq k)$, let $x_{d, w}{ }^{(0)}$ denote a representative from the unique cohomology class in $H C^{d-1, w}\left(A_{+}(k)\right)$. It is easy to see that the vectors of highest exterior degree (degree $\left.k n^{2}\right)$ in $\wedge\left(\mathrm{gl}_{n}(\mathbb{C}) \otimes A\right.$ ) represent a nonzero Lie algebra cohomology class. Also,

$$
\mathfrak{C}_{n}=\wedge x_{d, w}{ }^{(0)},
$$

has exterior degree $k n^{2}$. So by the usual properties of an exterior algebra, the theorem is proved if we show that $\mathfrak{C}_{n}$ is nonzero.

Let $\delta_{C}{ }^{+}$and $\delta_{C}{ }^{-}$be the duals of the maps $\partial_{C}{ }^{+}$and $\partial_{C}{ }^{-}$defined above. Let $\left\{E^{r}, \partial_{r}\right\}$ be the spectral sequence with $E^{2}$ term

$$
E^{2}=H^{*}\left(H C\left(A_{+}(k)\right), \delta_{C}{ }^{-}\right),
$$

which abutts to $H C(A(k))$. This spectral sequence provides a degree-preserving isomorphism between $H C\left(A_{+}(k)\right)$ and $H C(A(k))$, since we have already shown that $H C\left(A_{+}(k)\right)$ and $H C(A(k))$ have the same dimension. Under this isomorphism, $x_{d, w}{ }^{(0)}$ becomes a representative $y_{d, w}$ for a cyclic cohomology class in $H C(A(k))$. We write this representative as

$$
y_{d, w}=x_{d, w}{ }^{(0)}+x_{d, w}{ }^{(1)}+x_{d, w}{ }^{(2)}+\ldots,
$$

where $x_{d, w}{ }^{(i)}$ has degree $d$ and weight $w+(k+1) i$.

By Proposition 4.5 and Lemma 4.6 we have that $\wedge\left(\Phi_{n}\left(y_{d, w}\right)\right)$ is a nonzero element of $\wedge\left(\mathrm{gl}_{n}(\mathbb{C}) \otimes A(k)\right)$. Writing this out in terms of the $x_{d, w}{ }^{(i)}$ we have

$$
0 \neq\left(\wedge \Phi_{n}\left(x_{d, w}{ }^{(0)}\right)\right)+H,
$$

where $H$ is a sum of terms each having weight greater than the weight of $\wedge \Phi_{n}\left(x_{d, w}{ }^{(0)}\right)$. Note that

$$
\begin{aligned}
w t\left(\wedge \Phi_{n}\left(x_{d, w}{ }^{(0)}\right)\right. & =\sum \sum((k+1) d+t) \\
& =\{k(k+1)(n(n-1) / 2)\}+\{n k(k+1) / 2\} \\
& =n^{2} k(k+1) / 2 .
\end{aligned}
$$

But $n^{2} k(k+1) / 2$ is the highest weight occurring in $\wedge\left(\mathrm{gl}_{n}(\mathbb{C}) \otimes\left(A_{+}(k)\right)\right.$ so $H=0$. Thus $\mathfrak{C}_{n}$ is nonzero as desired. 
We next prove 2). Assume $\Phi_{n}$ is onto and let $\delta_{n}{ }^{+}$and $\delta_{n}$ be the coboundaries in the Koszul complexes for computing $H L\left(\mathrm{gl}_{n}(\mathbb{C}) \otimes A_{+}(k)\right)$ and $H L\left(\mathrm{gl}_{n}(\mathbb{C}) \otimes A(k)\right)$ respectively. Let $\delta_{n}{ }^{-}=\delta_{n}-\delta_{n}{ }^{+}$so $\delta_{n}{ }^{-}$increases degree by 1 and decreases weight by $k+1$.

As in the stable $(n=\infty)$ case, there is a spectral sequence $E_{p, q}^{r}$ with $E^{1}$ term $H L\left(\mathrm{gl}_{n}(\mathbb{C}) \otimes A_{+}(k)\right)$ which abutts to $H L\left(\mathrm{gl}_{n}(\mathbb{C}) \otimes A(k)\right)$. In our proof of statement $1)$, we showed that $\Phi_{n}(E(n))$ is mapped isomorphically onto $H L\left(\mathrm{gl}_{n}(\mathbb{C}) \otimes A(k)\right)$ by this spectral sequence. Let $\Phi_{n}^{r}(E(n))$ be the image of $\Phi_{n}(E(n))$ in $E^{r}$, and let $\delta^{r}$ denote the coboundary in $E^{r}$. Thus,
(A) $\delta^{r}: E_{p, q}^{r} \rightarrow E_{p+r, q-r(k+1)}^{r}$,
(B) $\delta^{r}\left(\Phi_{n}^{r}(E(n))=0\right.$,
(C) $\Phi_{n}^{r}(E(n)) \cap \operatorname{Im}\left(\delta^{r}\right)=0$.

We need to show that $\Phi_{n}(D(n)) \subset \Phi_{n}(E(n))$. Clearly it is enough to show that $\Phi_{n}(X) \in \Phi_{n}(E(n))$ for each generator $X$ of $D(n)$. Assume to the contrary that $\Phi_{n}(X)$ is not in $\Phi_{n}(E(n))$ for some generator. Choose $X$ to be a generator of $D(n)$ of minimal degree for which this happens, and let $q$ denote the weight of $\Phi_{n}(X)$.

By Proposition 4.5, $\Phi_{n}(X)$ vanishes at some point in the spectral sequence. Let $r$ be that point and let $\Phi_{n}^{r}(X)$ be the image of $\Phi_{n}(X)$ in $E^{r}$. Either $\delta^{r}\left(\Phi_{n}^{r}(X)\right)$ is nonzero or $\Phi_{n}^{r}(X)$ is in the image of $\delta^{r}$. In the latter case, $\Phi_{n}^{r}(X)=\delta^{r}(Y)$ for some $Y \in E_{p-r, q+r(k+1)}^{r}$. By the minimality of the degree $p, E_{p-r, q+r(k+1)}^{r} \subset \Phi_{n}^{r}(E(n))$ and so this case is impossible by observation $(B)$ above.

So it must be that $\delta^{r}\left(\Phi_{n}^{r}(X)\right)$ is a nonzero element of $E_{p+r, q-r(k+1)}^{r}$.

Claim. $E_{p+r, q-r(k+1)}^{r} C \Phi_{n}^{r}(E(n))$.

Proof. Clearly it is enough to show that

$$
E_{p+r, q-r(k+1)}^{1} C \Phi_{n}{ }^{1}(E(n))=\Phi_{n}(E(n)) .
$$

Suppose that this is false. Then there exists a nonzero $Y$,

$$
Y=\Phi_{n}\left(\Gamma_{1}\right) \wedge \ldots \wedge \Phi_{n}\left(\Gamma_{l}\right)
$$

in $H L^{p+r, q-r(k+1)}\left(\mathrm{gl}_{n}(\mathbb{C}) \otimes A_{+}(k)\right)$ with the $\Gamma_{i}$ generators of $H R C\left(A_{+}(k)\right)$ and with $\Phi_{n}\left(\Gamma_{1}\right)$ not in $\Phi_{n}(E(n))$. Let $p_{i}$ and $q_{i}$ be the degrees and weights of the $\Phi_{n}\left(\Gamma_{i}\right)$. We will use the observation that if $\Gamma$ is any generator of $H R C\left(A_{+}(k)\right)$ of degree $p^{\prime}$ and nonzero weight $q^{\prime}$ then

$$
-(k-1) / 2 \leqq\left((k+1) p^{\prime} / 2\right)-q^{\prime} \leqq(k-1) / 2 .
$$

By the minimality of $p$, we have $p_{1} \geqq p$ hence $q_{1}=0$ or $q_{1} \geqq((k+1) p-((k-1) / 2))$. Note that $q_{1}=0$ is impossible by Proposition 4.5 and the bound $p_{1} \geqq p \geqq(2 n+1)$. So,

$$
q_{1} \geqq(1 / 2)((k+1) p-(k-1)) .
$$

Also, $q \leqq(1 / 2)((k+1) p+(k-1))$ so

$$
q-r(k+1) \leqq q-(k+1)<(1 / 2)((k+1) p-(k-1)) \leqq q_{1} .
$$

But $q-r(k+1)=\sum q_{i}$ and this is a contradiction which proves the claim. 
Continuing with the proof of Theorem 4.9 we now have that $\Phi_{n}^{r}(E(n)) \cap \operatorname{Im}\left(\delta^{r}\right)$ is nonzero which is a contradiction.

The importance of Theorem 4.9 is that it shows that the image under $\phi_{n}$ of the subalgebra $E(n)$ is an exterior algebra with $n k$ generators of exactly the degrees and weights which are conjectured to be the degrees and weights of generators for $H L\left(\mathrm{gl}_{n}(\mathbb{C}) \otimes A_{+}(k)\right)^{g \mathbf{l}_{n}(\mathbb{C})}$.

The author believes that statement 2) of Theorem 4.9 is too weak. He conjectures that $\Phi_{n}(D(n))$ is zero with no assumptions about $\Phi_{n}$ being onto. One way to prove this would be to show that $\Phi_{n}(x)$ is in the image of $\delta_{n}{ }^{+}$for the explicit cyclic cohomology representatives $x$ of degree greater that $2 n$ given in Sect. 3. One might hope that for these $x, \Phi_{n}(x)$ would be zero in $\wedge\left(\mathrm{gl}_{n}(\mathbb{C}) \otimes A_{+}(k)\right)$. However, for $n=2$ and $k=2$, the representative $x$ of degree 5 and weight 8 given in Sect. 3 is the only possible cyclic cohomology representative and $\Phi_{2}(x)$ is nonzero in $\wedge\left(\mathrm{gl}_{2}(\mathbb{C}) \otimes A_{+}(2)\right)$ (although $\Phi_{2}(x)$ is zero in $H L\left(\mathrm{gl}_{2}(\mathbb{C}) \otimes A_{+}(2)\right)$ ).

\section{Part II - Dihedral cohomology}

In this part we prove results analogous to Theorem 4.9 for the symplectic and odd orthogonal Lie algebras. The overall approach is exactly the same as in Part I. First we extend $H D(A)$ to a ring $H R D(A)$ and define a degree and weightpreserving homomorphism $\Psi_{n}$ from $H R D(A)$ into $H L\left(\mathscr{G}_{n} \otimes A\right)$ (where $\mathscr{G}_{n}=o_{2 n+1}(\mathbb{C})$ or $\mathrm{sp}_{2 n}(\mathbb{C})$. The analogue of Proposition 4.5 is equivalent to a wellknown topological result. It is used to obtain the analogue of Theorem 4.9.

Most of the details will be just as in Part I and will therefore be omitted. Also the exposition is nearly identical for the orthogonal and symplectic cases and so we consider just the case of the symplectic Lie algebras. Recall that $\mathrm{sp}_{2 n}(\mathbb{C})$ is the Lie algebra of $2 n$ by $2 n$ real matrices $x$,

$$
x=\left[\begin{array}{l|l}
\frac{m_{1,1}}{m_{2,1}} & m_{1,2} \\
m_{2,2}
\end{array}\right],
$$

which satisfy $m_{1,2}{ }^{t}=m_{1,2}, m_{2,1}{ }^{t}=m_{2,1}$, and $m_{1,1}{ }^{t}=-m_{2,2}$. In order to define $\Psi_{n}$ we need notation for a basis of $\operatorname{sp}_{2 n}(\mathbb{C})$. This basis, denoted $\left\{t_{i, j}\right\}$ is given in the list below:

Basis vector

$$
\begin{aligned}
& \left(\begin{array}{c|c}
z_{i, j} & 0 \\
\hline 0 & -z_{i, j}
\end{array}\right) \\
& \left(\begin{array}{c|c}
0 & z_{i, j}+z_{j, i} \\
\hline 0 & 0
\end{array}\right) \\
& \left(\begin{array}{c|c}
0 & 0 \\
\hline z_{i, j}+z_{j, i} & 0
\end{array}\right)
\end{aligned}
$$

Notation

$$
\begin{gathered}
t_{i,-j}=t_{-j, i} \\
t_{i, j}=t_{j, i}
\end{gathered}
$$$$
t_{-i,-j}=t_{-j,-i}
$$ 
Definition 4.10. Define $\Psi_{n}: D\left(A^{*}\right) \rightarrow \wedge\left(\left(\mathrm{sp}_{2 n}(\mathbb{C}) \otimes A\right)^{*}\right)^{\mathrm{sp} 2 n(\mathbb{C})}$ by

$$
\Psi_{n}\left(L_{d}\left(a_{1}{ }^{*} \otimes \ldots \otimes a_{d}{ }^{*}\right)\right)=\sum\left(t_{i_{1}, i_{2}} \otimes a_{1}\right)^{*} \wedge \ldots \wedge\left(t_{i_{d}, i_{1}} \otimes a_{d}\right)^{*},
$$

where the sum is over all nonzero $i_{1}, \ldots, i_{d}$ with $-n \leqq i_{j} \leqq n$.

As with $\phi_{n}$, the definition of $\Psi_{n}$ seemingly depends on the order of $a_{1}, \ldots, a_{d}$. However it is easy to check that

1) $\Psi_{n}\left(L_{d}\left(a_{2}{ }^{*} \otimes \ldots \otimes a_{d}{ }^{*} \otimes a_{1}{ }^{*}\right)\right)=(-1)^{d-1} \Psi_{n}\left(L_{d}\left(a_{1}{ }^{*} \otimes \ldots \otimes a_{d}{ }^{*}\right)\right)$

2) $\Psi_{n}\left(L_{d}\left(a_{1}^{*} \otimes a_{d}{ }^{*} \otimes \ldots \otimes a_{2}{ }^{*}\right)\right)=(-1)^{(d-1)(d-2) / 2} \Psi_{n}\left(L_{d}\left(a_{1}{ }^{*} \otimes \ldots \otimes a_{d}{ }^{*}\right)\right)$.

It is also easy to check that $\Psi_{n}\left(L_{d}\left(a_{1}{ }^{*} \otimes \ldots \otimes a_{d}{ }^{*}\right)\right)$ is annihilated by sp ${ }_{2 n}(\mathbb{C})$ so $\Psi_{n}$ is a well-defined map from $D\left(A^{*}\right)$ into $\wedge\left(\left(\mathrm{sp}_{2 n}(\mathbb{C}) \otimes A\right)^{*}\right)^{\mathrm{sp}_{2 n}(\mathbb{C})}$.

As in Part I we define a bigraded ring $R D\left(A^{*}\right)$ to be $T\left(D\left(A^{*}\right)\right)$ modulo the relations

$$
\alpha \otimes \beta=(-1)^{(r+1)(s+1)} \beta \otimes \alpha,
$$

for $\alpha \in D^{r}\left(A^{*}\right)$ and $\beta \in D^{s}\left(A^{*}\right)$. The bigrading is that inherited from $D\left(A^{*}\right)$ but with degree shifted up by 1 . Extend $\delta_{D}$ to an antidetivation on $R D\left(A^{*}\right)$ and let $H R D\left(A^{*}\right)$ be the bigraded cohomology of the complex $\left(R D\left(A^{*}\right), \delta_{D}\right)$. The analogues of Proposition 4.2 and Theorem 4.4 hold with the role of $\mathrm{gl}_{n}(\mathbb{C})$ being played by $\mathrm{sp}_{2 n}(\mathbb{C})$ in Theorem 4.4 .

We will need the appropriate analogue of Proposition 4.5. Via deRham cohomology, the following result is equivalent to the well-known topological result that the cohomology of $\operatorname{Sp}(2 n, \mathbb{R})$ projects onto the cohomology of $\mathrm{Sp}(2 n-2, \mathbb{R})$ (see for example [23], p. 341).

Proposition 4.11. For each $s$, let $g(s)$ be the unique non-vanishing dihedral cohomology class in $H D^{4 s-2}(\mathbb{C})$. Let $G(n)$ and $F(n)$ be the subalgebras of $H R D(\mathbb{C})$ generated by $\{g(s): s \leqq 4 n\}$ and $\{g(s): s>4 n\}$ respectively. Then $\Psi_{n}(F(n))=0$ and $\Psi_{n}$ restricted to $G(n)$ is an isomorphism of $G(n)$ onto $H L\left(\operatorname{sp}_{2 n}(\mathbb{C})\right)$.

From this point on, the proof is identical to the proof given in Part I. We state the analogue of Theorem 4.9 and leave details of the proof to the reader.

Theorem 4.12. Let $A=A_{+}(k)$ and let $E(n)$ be the exterior algebra spanned by the $n k$ generators of $H R D(A)$ of degree less than $4 n$. Then $\Psi_{n}$ maps $E(n)$ isomorphically into $H L\left(\mathrm{sp}_{2 n}(\mathbb{C}) \otimes A\right)^{\mathrm{sp}_{2 n}(\mathbb{C})}$.

Lastly we need to discuss the case of the even orthogonal groups $o_{2 n}(\mathbb{C})$. In this case, the analysis above shows that the projection from the $n=\infty$ complex is not onto. The image is an exterior algebra with $(k+1)$ generators in each of the degrees $3,7,11, \ldots, 4 n-7$. As usual, one generator of degree $4 s+3$ has weight 0 and the remainder have weight $(k+1)(2 s+1)+t$ for $t=1,2, \ldots, k$. Thus $k+1$ generators of our conjectured cohomology ring are not in the image of the projection from $n=\infty$.

It is possible to write down cohomology representatives for these $k+1$ "missing" cohomology classes and show that they, together with the $(n-1)(k+1)$ generators projected from the $n=\infty$ complex, generate an exterior algebra with 
the degrees and weights predicted by the strong Macdonald conjectures. This requires detailed analysis of the centralizer algebra of $\operatorname{SO}(2 n, \mathbb{R})$ in the tensor powers of the defining representation (see [1] or [11]). This will appear in a separate note.

Acknowledgement. The author would like to thank Professor J.-L. Loday for many helpful suggestions during the preparation of this manuscript.

\section{References}

1. Brauer, R.: On algebras which are connected with the semisimple continuous groups. Ann. Math. 38, 857-872 (1937)

2. Bressoud, D., Zeilberger, D.: A proof of Andrews' $q$-Dyson conjecture. Discrete Math 54, 201-224 (1985)

3. Cartan, E.: Sur les invariants integraux de certains espaces homogenes clos. Ann. Soc. Math. Pol. 8, 181-225 (1929)

4. Chevalley, C., Eilenberg, S.: Cohomology theory of Lie groups and Lie algebras. Trans. Am. Math. Soc. 63, 85-124 (1948)

5. Connes, A.: Non commutative differential geometry, Part I, The Chern character in $K$-homology (preprint IHES 1982)

6. Connes, A.: Non commutative differential geometry, Part II, DeRham homology and noncommutative algebra (preprint IHES 1983)

7. Garland, H., Lepowsky, J.: Lie algebra homology and the Macdonald-Kac formulas. Invent. Math. 34, 37-76 (1976)

8. Goodwillie, T.: Cyclic homology, derivations and the free loop space. Topology 24, 187-215(1985)

9. Guichardet, A.: Cohomologie des groupes topologique et algèbres de Lie. Textes Mathématiques, CEDIC/Fernand Nathan (1980)

10. Hanlon, P.: The proof of a limiting case of Macdonald's root system conjectures. Proc. Lond. Math. Soc. 49, 170-182 (1984)

11. Hanlon, P.: On the decomposition of the tensor algebra of the classical Lie algebras. Adv. Math. 56, 238-282 (1985)

12. Hopf, H.: Über die Topologie der Gruppen-Mannigfaltigkeiten. Ann. Math. 42, 22-52 (1941)

13. Kostant, B.: private communication

14. Kostant, B.: A theorem of Frobenius, a theorem of Amitsur-Levitsky and cohomology theory. J. Math. Mech. 7, 237-264 (1958)

15. Koszul, J.L.: Homologie et cohomologie des algèbres de Lie. Bull. Soc. Math. Fr. 78, 65-127 (1950)

16. Loday, J.-L., Quillen, D.: Cyclic homology and the Lie algebra homology of matrices. Commun. Math. Helv, 59, 565-591 (1984)

17. Loday, J.-L.: Homologies diedrale et quaternionique. Adv. Math. (to appear)

18. Loday, J.-L., Procesi, C.: Homology of orthogonal and symplectic matrices (preprint)

19. Macdonald, I.G.: Some conjectures for root systems. SIAM J. Math. Anal. 13, 988-1007 (1982)

20. Macdonald, I.G.: Affine root systems and Dedekind's $\eta$-function. Invent, Math. 15, 91-143 (1972)

21. Regev, A.: unpublished manuscript

22. Tsygan, B.L.: Homology of matrix algebras over rings and the Hochschild homology. (in Russian), Usp. Mat. Nauk. 38, 217-218 (1983)

23. Whitehead, G.: Elements of homotopy theory. Berlin-Heidelberg-New York: Springer 1978 